\title{
TDU-Unterhaltungsindex
}

\section{Ein Instrument zur empirischen Ermittlung von Unterhaltungserleben}

\author{
Werner Früh / Carsten Wünsch / Pascal Klopp
}

Zur Erfassung von Unterhaltungserleben wird ein Messinstrument entwickelt und in drei empirischen Untersuchungen validiert. Es geht zurück auf die "triadisch-dynamische Unterhaltungstheorie" (Früh 2002 \& 2003a). Diese verstebt Unterhaltungserleben als ein emotionsähnliches Erleben (Makroemotion), welches durch eine tendenziell positive Valenz, eine prinzipielle Beherrschbarkeit der Konsequenzen der Rezeption und die Nutzung allgemeiner Dispositionsfreibeiten (Souveränität/Kontrolle) gekennzeichnet ist. Das Messinstrument kann Unterhaltung anhand dieser Aspekte als ein stetiges Merkmal in beliebigen Kontexten erfassen (also z. B. auch bei der Nachrichtenrezeption). In den Validierungsstudien wird neben Reliabilität, Objektivität und Facevalidität insbesondere die Konstruktvalidität geprüft. Die Ergebnisse bestätigen unsere Erwartungen. Sie zeigen, dass das vorgestellte Instrument zu anderen und (aus unserer theoretischen Perspektive) besseren Messergebnissen führt, als bekannte Verfabren der Unterhaltungsmessung.

Keywords: Unterhaltung, Unterhaltungerleben, empirische Methoden

\section{Theorie}

Auf der Grundlage des dynamisch-transaktionalen Ansatzes (DTA) mit seinen Bestandteilen molarer Kontext, Transaktion und Dynamik entwickelte Früh die „triadisch-dynamische Unterhaltungstheorie“ (TDU) (Früh 2002, 2003a). Hier werden wir in einem ersten Schritt eine Beschreibung des Konstrukts „Unterhaltung“ aus der TDU ableiten und einige Differenzierungen und Spezifikationen vornehmen, mit dem Ziel, anschließend ein Messinstrument vorzustellen, das die statische Variante des in der TDU entwickelten Unterhaltungsbegriffs operationalisiert. ${ }^{1}$ In einem dritten Schritt werden dann die Ergebnisse mehrerer Studien zur Validierung dieses Messinstruments herangezogen. Die folgenden theoretischen Ausführungen sind weder eine Kurzfassung der Theorie noch sollen darin theoretische Probleme diskutiert werden. Dazu verweisen wir auf die Originalpublikationen (Früh 2002, 2003a). Hier werden nur die im Messinstrument benutzten Aspekte noch einmal kurz zusammengestellt und definiert.

Unterhaltung ist als spezifisches kognitiv-affektives Erleben nicht direkt beobachtbar und muss deshalb über Indikatoren erschlossen werden. Dies geschieht meist über die Selbstauskunft der Personen, seltener mittels Beobachtung von Mimik oder Gestik. Wenn - wie noch gezeigt wird - eine begründete Vermutung besteht, dass die sprachliche Externalisierung der Begriffsbedeutung mehrdeutig ist, kann man als Forscher nicht einfach den Zuschauer fragen: „Haben sie sich unterhalten?” Niemand kann genau sagen, ob Rezipienten dann, wenn sie von Unterhaltung sprechen, erstens alle dieselbe Vorstellung damit verbinden (z. B. bestimmte Objektklassen wie Spielfilme etc.; be-

1 Die dynamische Variante erfordert mehr Aufwand bei der Erfassung und ist deshalb für eine schnelle praktische Anwendung weniger geeignet. 
stimmte Tätigkeiten wie z. B. spielen oder Musik hören oder aber eine Emotion) und wenn sie eine Emotion meinen, ob es sich dabei zweitens auch bei allen um ein und dieselbe Emotion handelt. Deshalb ist dieser Indikator nicht falsch, aber ungenauer und unsicherer als das hier vorgeschlagene etwas differenziertere Erhebungsinstrument. Es löst zwar nicht grundsätzlich das genannte Problem des indirekten Zugangs, geht aber etwas subtiler damit um und benutzt einen theoretisch und operational weiter präzisierten Unterhaltungsbegriff.

In der TDU wird das Konstrukt „Unterhaltung“ als ein allgemeiner Erlebensmodus ${ }^{2}$ beschrieben, der sich überall, zu jeder Zeit und bei allen Personen zeigen kann. Unterhaltung ist keine bestimmte, allein gegenstandsabhängige Tätigkeit, sondern eine generelle „Kulturtechnik“ (Schmidt 2003: 326). Angesichts dieser diffusen „Entgrenzung“ des Gegenstandes stellt sich die Frage nach den spezifischen Bedingungen, unter denen Unterhaltung entsteht. Früh nennt unter Rückgriff auf die Heuristik des „molaren Kontextes“ keine fixen Voraussetzungen, sondern eine variable Faktorenkonstellation, die bei einer geeigneten „Passung“ Unterhaltung möglich macht. Sie wird als „triadisches Fitting " bezeichnet, da es sich um die Passung der drei Dimensionen Objekt/Stimulus, Person und situativer bzw. gesellschaftlicher Kontext handelt. Das Unterhaltungserleben gemäß der TDU ist in seinen grundlegenden Merkmalen zunächst gegenstands-, personen- und situationsunabhängig als allgemeines Phänomen konzipiert und dann anhand der TV-Rezeption konkretisiert. Insofern ist der Geltungsbereich der TDU derzeit auf audiovisuelle Unterhaltung begrenzt, wobei auch interaktive Formen (Videospiele etc.) eingeschlossen sind. Fast alle hier vorzustellenden Operationalisierungen beziehen sich primär auf audiovisuell stimuliertes Unterhaltungserleben. Ungeprüft, jedoch von der Anlage her mit vermutlich nur geringen Modifikationen möglich, wird sich die TDU aber mindestens auf alle medieninduzierten Unterhaltungsformen, möglicherweise sogar auch auf nichtmediale Unterhaltung übertragen lassen. ${ }^{3}$

Die Theorie in Stichworten:

Auf der Grundlage eines dynamischen Energiebudget-Managements entsteht Unterhaltung als Makroemotion, ${ }^{4}$ die in ihrer Spezifität zwischen allgemeiner Primäremotion

2 Im Folgenden verwenden wir im Zusammenhang mit Unterhaltung verschiedene Begriffe, welche alle das gleiche von uns gemeinte Konstrukt aus unterschiedlicher Perspektive (z. B. als Prozess, dessen Resultat oder abstrakt kategorisierend ) bezeichnen. Als Rezeptionshandlung führt eine charakteristische Art der Informationsverarbeitung (Rezeptionsmodus) auf der Metaebene des Verarbeitungsprozesses zu einer Makroemotion. Da sie transaktional verbundene kognitive und emotionale Bestandteile enthält, kann man die Makroemotion auch als eine bestimmte Art kognitiv-affektiven Erlebens bezeichnen. Spezifiziert man ihren Geltungsbereich bzw. Entstehungshorizont, dann ist sie eine allgemeine Modalität der Informationsverarbeitung, die jederzeit, in allen Situationen, bei allen Stimuli (sogar internalen) etc. entstehen kann, wenn die Komponenten in einer günstigen Konstellation zueinander passen (s. „triadisches Fitting“).

3 Der Grund liegt darin, dass die Theorie am individuellen Erleben ansetzt, das durch beliebige Variationen der triadischen Komponenten entstehen kann. Externe Stimuli müssen also nicht von Medien kommen, und selbst internale Stimulationen ganz ohne äußeren Anlass sind theoriekonform.

4 Makroebene/Makrostruktur/Makroemotion: Menschen verarbeiten Textinformation (bzw. allgemein das Medienangebot) ,automatisch“ so, dass sie einerseits nicht alle Informationen Wort für Wort auswendig lernen müssen, andererseits aber auch das aus ihrer Sicht Wichtigste so auswählen und integrieren, dass daraus eine kohärente, subjektiv stimmige Bedeutungseinheit entsteht, die van Dijk allgemein „Makrostruktur“ nennt (Van Dijk 1980: 41ff.). Er bezieht dieses 
und spezifischer Emotion liegt. Sie konstituiert sich in einem ersten Schritt als transaktional gekoppelte, also simultan entstehende Kognition und Primäremotion. Durch kognitive Kontrollprozesse (appraisal) in konkreten Situationen und hinsichtlich eines konkreten Fernsehangebots werden dann die allgemeinen Unterhaltungspotenziale dreier Gruppen von Bedingungsfaktoren durch den Rezipienten interpretiert und hinsichtlich ihrer Zweckdienlichkeit für Unterhaltung geprüft: Medienangebot, personale (d. h. eigene) Dispositionen und sozialer/situativer Kontext. Im dritten Schritt, der sich über die ganze Rezeptionsphase erstreckt, wird dann kontinuierlich festgestellt, ob die vorgefundenen Bedingungsfaktoren aller drei Merkmalsgruppen so zueinander passen, dass sie Unterhaltung (mehr oder weniger optimal und umfangreich) ermöglichen. Durch eine transaktional integrierte Informations- und Emotionsverarbeitung werden die einzelnen kognitiv-affektiven Wahrnehmungen auf der Mikroebene sukzessiv zu einer allgemeineren Makroemotion transformiert. Während auf der Mikroebene alle denkbaren spezifischen Emotionen positiver wie negativer Art erlebt werden können, findet das Unterhaltungserleben parallel dazu als tendenziell positive Emotion auf der Makroebene statt. Wesentliche Voraussetzungen für die Transformation zum positiven Erleben ist der Eindruck von Souveränität und Kontrolle sowie das momentane Fitting der personalen, medialen und sozialen/situativen Bedingungsfaktoren („triadisches Ursachenfitting “; Früh 2002: 142ff.). Emotionen und Stimmungen verstehen wir dabei als unterschiedliche Phasen eines einheitlichen Prozesses, in dem Unterhaltungsemotionen fließend in Stimmungen übergehen, wie auch umgekehrt Stimmungen zu Emotionen kontraktieren können.

Unterhaltung ist also eine allgemeine Modalität der Informationsverarbeitung, mit der beliebige Inhalte entsprechend transformiert werden können. Auf das Fernsehen bezogen kann Unterhaltung damit generell bei allen Fernsehangeboten entstehen. Definitionsbedürftig ist, welche Kriterien hierfür relevant sind und welche Faktoren dies bewirken.

Zusammengefasst ist Unterhaltung also ein

a) tendenziell positives kognitiv-affektives Erleben,

b) das auf der Makroebene

c) im Rahmen einer geeigneten Bedingungskonstellation entsteht und

d) Souveränität/Kontrolle voraussetzt.

Es kann vor, während und nach der Rezeption entstehen.

Konstrukt auf das Generalthema von Texten, das als kohärente und verdichtete Bedeutungsstruktur bei der Lektüre entsteht. Es kann weitgehend auch auf die Emotionsrezeption übertragen werden (vgl. Früh 2002: 163ff.). Personen rezipieren Schritt für Schritt die einzelnen Informationen des Textes und formen schon sehr schnell eine globale Vorstellung seines Inhaltsund Emotionsgehaltes. In der Folge wird dann permanent geprüft, ob die weiteren Informationen auf der „Mikroebene“ zur Wahrnehmungshypothese passen. Dies lässt sich formal so darstellen, dass die bereits wahrgenommenen Emotionen auf der Mikroebene gemeinsam mit den Emotionserwartungen zu einem vorläufigen Gesamtbild kombiniert werden, wobei bei fortschreitender Rezeption der Anteil der Emotionserfahrungen immer größer, derjenige der Emotionserwartungen immer geringer wird. Die Emotionen auf der Mikroebene bleiben dabei jedoch nicht original erhalten, sondern sie gehen in verarbeiteter Form (selektiert, abstrahiert, elaboriert, integriert etc.) in die Makrostruktur ein. Ein den Makroemotionen teilweise ähnliches Konzept findet sich auch bei Oliver (1993): Sie beschreibt Meta-Stimmungen als durch eine distanzierte Bewertung (z. B. „Ich finde es schön, auch einmal traurig zu sein“) der „primären Stimmungen“ (z. B. Trauer) ausgelöstes (z. B. angenehmes) Erleben. 
Diese Kriterien sollen auf der Grundlage der TDU kurz erläutert werden (nähere Beschreibung siehe Früh 2002; 2003a).

Unterbaltung als spezifische positive Makroemotion: Erklärungsbedürftig ist insbesondere, wie sich Unterhaltung als positive Emotion von anderen positiven Emotionen unterscheidet und unter welchen Voraussetzungen der „switch“ zu Unterhaltung stattfinden kann. Weder der Gegenstandsbereich noch die Bandbreite der Emotionen, auf die sich Unterhaltung beziehen kann, ist beschränkt. Eine Eingrenzung gibt es zunächst nur dahingehend, dass Unterhaltung auf der Makroebene entsteht und dort ein tendenziell positives Erleben darstellt. Makroebene beschreibt gegenüber den unmittelbaren, linearen Textverarbeitungsprozessen ${ }^{5}$ (Wort für Wort, Szene für Szene etc.) eine allgemeinere und dynamische Repräsentation der inhaltlichen und emotionalen Textbedeutung. Sie hat bereits kurz nach Rezeptionsbeginn ganzheitlichen Charakter (holistisches mentales Modell; siehe auch FN 2; Früh 2002: 160ff.). „Tendenziell“ meint, dass die positive Tönung erstens als dominantes Verarbeitungsprodukt vieler verschiedener Emotionen auch negative oder kategorial andere „Beimischungen“ enthalten kann und dass sie zweitens, zusätzlich oder anstatt, auch erst sekundär infolge elaborativer „Kommentierungen“ (Früh 2002: 128 ff.; Früh 2003a: 43ff.) entstehen kann (z. B. im Sinne von „Wie schön ist es doch, melancholisch zu sein!“ oder „Angstlust“ etc.). Damit beschreibt die positive Valenz der Unterhaltung nicht nur eine hedonistische Erlebensqualität (auf einer Lust-Unlust-Dimension), sondern insgesamt eine attraktive und positive Wertigkeit, die über Hedonismus hinausgehend auch positive Gefühle beinhaltet, die z. B. auf Erfolg, Kontrolle, Erkenntnisgewinn oder Bewertungen beruhen.

Dies reicht jedoch noch immer nicht aus, um Unterhaltung eindeutig zu bestimmen. Es gibt viele andere Emotionen positiver Art, welche dieselben Bedingungen erfüllen, aber keine Unterhaltung darstellen. Wenn ein Zuschauer z. B. beim Fernsehen erregt und engagiert verfolgt, wie die deutsche Fußball-Nationalmannschaft die Weltmeisterschaft erringt, kann er sich, je nach Einstellung, hervorragend unterhalten oder aber auch Stolz empfinden - möglicherweise auch beides. Wenn nun Unterhaltung ein originäres Empfinden darstellen soll, dann ist Stolz als andere spezifische Emotion nicht identisch mit Unterhaltung. Der Zuschauer kann beispielsweise mit der Überlegung, „das alles ist nur ein Spiel und ich gehöre selbst nicht der Nationalmannschaft an“, eine Art relativierten „als ob-Stolz“ empfinden, der als positiver Aspekt auf der Mikroebene (neben anderen) zu einer unterhaltenden Makroemotion verarbeitet wird, die durch den Bestandteil „Stolz“ nur eine bestimmte „Färbung“ erhält. Fehlt ihm jedoch diese Distanz vollständig, weil er sich völlig mit der Mannschaft identifiziert, dann wird der Stolz zum „nationalistischen Ernstfall“. Der Zuschauer hat dann zwar immer noch ein positives Gefühl, was aber keine Unterhaltung darstellt.

Was bringt nun aber eine Person dazu, gleiche Wahrnehmungen unterschiedlich, entweder speziell als Unterhaltung oder aber als andere positive Emotion wahrzunehmen? Früh argumentiert, dass dafür spezifische Kontrollprozesse verantwortlich seien. Insbesondere der Faktor „Souveränität/Kontrolle“ spielt eine besondere Rolle, um Unterhaltung zu ermöglichen und von anderen positiven Emotionen zu unterscheiden. 


\subsection{Souveränität/Kontrolle}

Souveränität/Kontrolle hat die Funktion, den unterhaltungsspezifizierenden Interpretationsmodus zu prägen oder gar zu erzeugen (Früh 2002: 108ff.; 128-140; Früh 2003a: 31ff.). Sie steuert einen Informations- und Emotionsbestandteil bei, der als charakteristischer Akzent in jedes Unterhaltungserleben eingeht. Er wird dabei nicht nur postrezeptiv nachweisbar sein, sondern auch bereits die Informations- und Emotionsverarbeitungsprozesse während der Rezeption dynamisch mitprägen (Früh 2002: 222). Keine einzige spezifische positive Emotion ist per se Unterhaltung, aber jede davon oder alle zusammen können unter dem beschriebenen Kontrollmodus zu mehr oder weniger dominanten oder gar alleinigen Bestandteilen von Unterhaltung werden, die jedoch dadurch einen charakteristischen Akzent erhalten. Durch diesen Bedeutungsakzent mutiert beliebiges Erleben zu Unterhaltungserleben.

Allerdings geschieht dies in unterschiedlichem Maße. Früh konzipiert Unterhaltung als unabhängige, stetige Variable. Es gibt also keinen quantitativen Schwellenwert, bei dem Nicht-Unterhaltung in Unterhaltung umschlägt, vielmehr kann sie als mehr oder weniger umfangreiche Beimischung mit anderen Erlebensweisen und rezeptiven Zielsetzungen auftreten oder aber die dominante Rezeptionsweise darstellen.

Die beiden von uns stets parallel gebrauchten ${ }^{6}$ Begriffe Souveränität und Kontrolle überschneiden sich nur teilweise in ihrer Bedeutung, jeder enthält auch einen eigenständigen Bedeutungsanteil. Während sich Kontrolle insbesondere auf die Überschaubarkeit und Beherrschbarkeit der Konsequenzen unterhaltender Rezeption bezieht, betont Souveränität stärker die Dispositions- und Entscheidungsfreiheit des Rezipienten (Früh 2002: 145ff.). Beide Kriterien können in einigen Fällen auch konkurrieren. Souveränität kann beispielsweise einen Kontrollaspekt wie „konsequenzenlos für den Lebensalltag“ einschränken, indem der Rezipient souverän entscheidet, dass er bestimmte positive oder negative Konsequenzen für die reale Lebenswelt nicht vermeiden, sondern im Gegenteil sogar gezielt hervorrufen und nutzen (z. B. lernen, Stärkung der Selbstsicherheit etc.), zumindest aber in Kauf nehmen möchte (z. B. Freizeitverbrauch, Auflösung sozialer Beziehungen, Erschöpfung, Schlafmangel, Ablenkung, Verzicht auf attraktive Handlungsalternativen etc.; vgl. z. B. Früh 2002: 148; 151f.; 184f.).

Allgemein kann man sagen, dass die erlebte Souveränität und Kontrolle (bzw. erlebte Kompetenz als Resultat einer erfolgreich ausgeübten Kontrolle) mit der Reduktion von tatsächlichen oder als möglich erachteten Restriktionen und dem Ausnutzungsgrad der damit gewonnenen Freiräume steigt. Unter Restriktionen sind jegliche „Zumutungen“ in Form von Anforderungen, Beschränkungen, Zwängen, Notwendigkeiten etc. $\mathrm{zu}$ verstehen, und das ist sehr generell und ausschließlich gemeint (auch die Abhängigkeit von der Schwerkraft stellt z. B. eine solche Restriktion oder „Zumutung“ dar). Sie können von der Alltagswelt (Konsequenzen für den Alltag), der Rezeptionssituation (restriktive Bedingungen der sozialen Situation), internalisierten Werten und Normen (Restriktionen des gesellschaftlichen/kulturellen Kontextes) und in der Fantasie durch jegliche „Herausforderung“ physikalischer oder logischer Art gegeben sein (Früh 2002: 123ff.; 151; 184ff.). Souveränität/Kontrolle besitzt also in Form von „Ab-

6 Im weiteren Verlauf des Textes wird zugunsten einer besseren Lesbarkeit z. T. auch nur noch der Begriff „Souveränität“ verwendet. Auch an diesen Stellen ist stets das Konstruktpaar „Souveränität/Kontrolle“ gemeint. 
wehr und Entgrenzung“ sowohl einen passiv-defensiven als auch einen aktiv-initiativen Aspekt ${ }^{7}$.

Passive Souveränität/Kontrolle: Sie wird dadurch ausgeübt, dass Restriktionen als externale oder internale Zumutungen ignoriert oder verdrängt bzw. Situationen gesucht werden, die möglichst wenige Restriktionen als Zumutungen enthalten. Sie sind dann in der aktuellen Situation kognitiv irrelevant, obwohl sie ggf. durchaus existent bleiben können (siehe auch unten das Beispiel für „ausblenden“).

Aus passiv-defensiver Perspektive ist Unterhaltung also umso ausgeprägter möglich, je irrelevanter solche Restriktionen sind bzw. erscheinen. Allein schon das Wissen um diesen Freiraum ist angenehm, d. h. das Bewusstsein der bloßen Option: „Ich könnte, wenn ich wollte ..." kann bereits ausreichen. Allerdings ist mit „Wissen“ nicht unbedingt eine permanente Fokussierung gemeint. Dies könnte als ablenkendes Moment eher unterhaltungsabträglich sein. Es genügt eine gelegentliche Vergewisserung und ansonsten ein permanenter Hintergrundcheck (Früh 2002: 144f.). Bemerkt der Rezipient „Zumutungen“ irgend einer Art, kann er sie zu reduzieren versuchen. Die Reduktion erfolgt kognitiv, indem die Restriktionen einfach „ausgeblendet“ werden; manipulativ wird sie dagegen durch Verändern oder Aufsuchen einer weniger restringierten Situation erreicht. So kann zum Beispiel der Rezipient, der sich durch eventuelle Blicke der Nachbarn durch das erleuchtete Fenster gestört fühlt und so in seiner (passiven) Souveränität eingeschränkt ist, entweder die Vorhänge zuziehen (manipulativ) oder aber die Existenz möglicher Beobachter verdrängen oder einfach vergessen (kognitiv). Wichtig ist nur, dass diese Restriktionen nicht als relevante Einflüsse im Fokus der Aufmerksamkeit stehen, obwohl sie ggf. durchaus existent bleiben.

Darüber hinaus erstreckt sich passive Souveränität/Kontrolle auf den Aspekt der „Interesselosigkeit“. Der Rezipient muss seine Tätigkeit überwiegend als intrinsisch motivierte, nicht fremdbestimmte Handlung begreifen. ${ }^{8}$ Sämtliche extrinsischen, außerhalb der Tätigkeit liegenden Motive und Handlungsziele stellen Zumutungen im oben vorgestellten Sinne dar und erschweren ein Unterhaltungserleben oder machen es gar unmöglich, sobald sie im Fokus der Aufmerksamkeit liegen (ein Rezipient kann z. B. durchaus ein Lehrbuch lesen um zu lernen und sich dennoch unterhalten, wenn er das Ziel des Lernens vergisst und sich bei der Lektüre des interessanten Textes einfach nur angenehm fühlt; Früh 2002: 169f.).

Solche tatsächlich eliminierte oder kognitiv kontrollierte (und somit während der Re-

7 Die Begriffe „passiv“ und „aktiv“ beziehen sich nicht auf die Aktivität von Personen, sondern die defensive oder offensive Funktion der Souveränität/Kontrolle. Passive Souveränität/Kontrolle meint das Fernhalten oder Ausblenden von „Zumutungen“, also eine defensive Haltung zur Schaffung einer geschützten Lebensweltnische. Innerhalb dieses Refugiums kann das Individuum dann wiederum aktiv werden, indem es die jetzt vorhandenen Möglichkeiten offensiv nutzt (aktive Souveränität/Kontrolle). Natürlich kann diese geschützte Lebensweltnische (also die passive Souveränität) auch aktiv - diesmal im Sinne von Aktionen des Individuums - herbeigeführt und manipuliert werden (siehe nächster Abschnitt). Die Funktion der aktiven Handlung bleibt dabei aber nach wie vor passiv-defensiv im Sinne einer Abwehr von „Zumutungen“.

8 Hier bestehen Ähnlichkeiten zum Konzept der Autotelik bei Csikszentmihalyi (2000) beim Flow-Erleben. Das Flow-Erleben unterscheidet sich allerdings deutlich vom hier beschriebenen Unterhaltungskonstrukt in der Rolle, welche die „Vermitteltheit“ bzw. das Bewusstsein dieser spielt (vgl. Früh 2002: 132f.). 
zeption mehr oder weniger irrelevante) Restriktionen und Zumutungen können dabei 1) aus einem Konflikt zwischen der rezipierten Textbedeutung und (internalisierten) gesellschaftlichen bzw. kulturellen Normen und Werten resultieren („gesellschaftlich-kultureller Kontext“). Einige dieser Normverletzungen durch den Text werden vor allem dann relevant, wenn die Rezeption in einer Gruppe erfolgt (z. B. Pornografie, Gewalt, Triviales). Andere Werte und Normen sind so zentral und so weit internalisiert, dass ihre Verletzung auch ohne soziale Kontrolle als starke Zumutung wirksam wird. Wieder andere stellen weniger Zumutungen als vielmehr Versuchungen zur Unterhaltung dar, denen der Rezipient aufgrund internalisierter Werte widersteht (vgl. Früh 2002: 129). 2) Eine weitere Quelle potenzieller Restriktionen und Zumutungen ist in der Relevanz des Textinhaltes für den Alltag und somit dessen Bedeutsamkeit über die Rezeptionssituation hinaus zu suchen („Alltagsrelevanz“). Ein Beispiel für eine solche Zumutung wäre eine Nachricht über den Konkurs des eigenen Arbeitgebers. Diese Nachricht stellt durch den wahrscheinlichen Verlust des Arbeitsplatzes eine starke Restriktion für die zukünftige Lebensplanung des Rezipienten dar. Wird sie auch als Restriktion wahrgenommen (es fällt schwer, diese Information als irrelevant zu ignorieren), so kann hier kein Unterhaltungserleben entstehen, obwohl dies ohne eigene Betroffenheit durch eine spektakuläre, dramatische journalistische Aufbereitung ggf. möglich gewesen wäre. ${ }^{9}$ 3) Die dritte Quelle von Restriktionen und Zumutungen ist im situativen Kontext der Rezeption und dessen „Unterhaltungstauglichkeit“ auszumachen („situativer Kontext“). Als Beispiele wären hier die Anwesenheit anderer (fremder) Personen (teilweise Überschneidung mit 1.) oder dringend erledigungsbedürftige Aufgaben zu nennen. Zu diesen Restriktionen gehören auch extrinsische oder außerhalb der unterhaltenden Tätigkeit liegende Handlungsmotive und Handlungsziele (wie das oben vorgestellte Beispiel des Lernens; vgl. auch Früh 2002: 112ff.).

Aktive Souveränität/Kontrolle: Voraussetzung für Unterhaltung ist auch zumindest ein Minimum an aktiver Souveränität, d. h. der Rezipient muss sich wenigstens ansatzweise auf die unterhaltende Rezeptionsmodalität „einlassen“ im Sinne von Involvement (Früh 2002: 134ff.; zu Involvement und verwandten Konstrukten siehe auch: Salmon 1986; Lombard \& Ditton 1997). Deshalb sprechen wir bisher auch immer nur davon, dass die genannten Faktoren Unterhaltung „ermöglichen“. Aktive Souveränität/Kontrolle besteht darin, den durch passive Souveränität geschaffenen Freiraum mehr oder weniger intensiv auszunutzen. Rezipienten können Kognitionen, Emotionen und konkrete Handlungen (meist in der Fantasie) realisieren, indem sie ungeachtet der „ansonsten" definitiv existenten Restriktionen in einer Weise kognitiv, emotional oder konativ agieren, wie es normalerweise nicht möglich ist bzw. mit hohen „Kosten“ verbunden wäre. Eine solche „Entgrenzung“ kann durch jedwede „Überwindung“ physikalischer, logischer und anderer Bedingungen in der Fantasie stattfinden, aber auch die involvierte und interessierte Auseinandersetzung mit einem realen und ernsten Thema sein, solange dies auf der Grundlage passiver Souveränität geschieht, also Restriktionen und Zu-

9 Damit ist nicht gemeint, dass eine Informationssendung abrupt zu einer Unterhaltungssendung mutieren müsste. Wie bereits dargelegt, fassen wir Unterhaltung als stetiges Merkmal auf, das auch als mehr oder weniger starke „Beimischung“ zu einem dominant anderen Erleben, wie z. B. die auf Wissenserwerb ausgerichtete Auseinandersetzung mit den Themen einer Bildungssendung, auftreten kann. 
mutungen weitgehend kontrolliert bzw. minimiert sind. So kann man z. B. in seiner Fantasie fliegen, folgenlos fremdgehen oder einen Eimer Sekt so lange leer trinken, bis er überläuft. Man kann aber auch eine neue, gerechtere Weltordnung einführen, in der alle Ungleichheits- und Hungerprobleme gelöst sind, jedenfalls solange es sich dabei nur um geistige „Ausflüge“ handelt, die keine Konsequenzen haben und aus denen man jederzeit aussteigen kann (die Weltordnung muss also nicht zu Ende gedacht werden und ihr Scheitern führt auch nicht zu realem Hunger) (vgl. Früh 2002: 123f.).

Ausgehend von dieser Beschreibung unterscheiden wir bei aktiver Souveränität eine kognitive und eine affektive Strategie. ${ }^{10}$ Aktive Souveränität kann ein Rezipient durch kognitive Aktivitäten erlangen, die auch als kognitives Involvement bezeichnet werden (z. B. Roser 1990): Er denkt mit, setzt sich mit dem Gesehenen kognitiv, analytisch, problemorientiert auseinander oder beschäftigt sich, angeregt durch das Gesehene, gedanklich mit analogen Dingen, schlussfolgert oder assoziiert andere Themen und Erinnerungen. „Affektiv“ kann er dies erreichen, indem er mitfühlt, (empathisch) das Gesehene miterlebt, oder indem er, angeregt durch das Gesehene, in fantasievollen und emotionsträchtigen „Tagträumen“ schwelgt oder einfach seine „Seele baumeln“ lässt (Früh 2002: 167ff.). Bei den Strategien lässt sich, wie bei der Beschreibung schon angedeutet, noch eine weitere Unterteilung treffen: Das Erreichen aktiver Souveränität kann sowohl bei der kognitiven als auch bei der affektiven Strategie eher stimulusgeleitet, durch Mitdenken bzw. Mitfühlen, vonstatten gehen (external) oder eher fantasiegeleitet bzw. elaborativ erfolgen (internal). In Abbildung 1 sind aktive und passive Souveränität mit ihren jeweiligen Subdimensionen dargestellt.

\section{Abbildung 1: Formale Struktur der Dimensionen von Unterhaltungserleben}

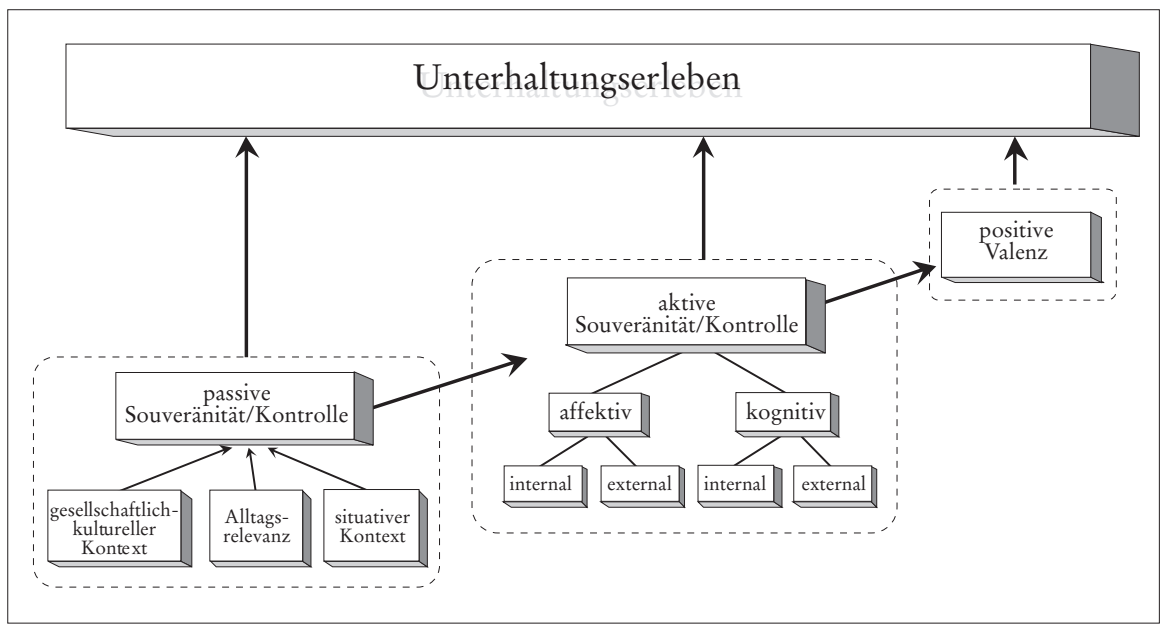

10 Beide Strategien sind empirisch kaum strikt trennbar (im Sinne des DTA werden Emotionen und Kognitionen als transaktional verbundenes Syndrom betrachtet; vgl. Früh 2002: 116ff.). Es werden wohl eher kognitiv-affektive Mischformen zu beobachten sein. 
Es gibt also vor allem in der Fantasie fast unbeschränkte Freiheiten, und das wissen die Zuschauer, aber viele von ihnen wollen alle Möglichkeiten vielleicht gar nicht ausschöpfen. Zur aktiven Souveränität gehört auch, dass der Rezipient es sich leistet, einfach nichts zu tun, schlimme Zumutungen zu ertragen oder die trivialsten und lächerlichsten Angebote amüsant und sogar schön zu finden; er ist niemand Rechenschaft schuldig, und es schadet auch nicht seinem Image. Der Rezipient bleibt also der Souverän, der - im Unterschied zum „normalen“ Leben - alles riskieren kann, der die Entscheidungen trifft und alles unter Kontrolle behält. Bereits dieses Bewusstsein der unbegrenzten Möglichkeiten und der Freiheit von allen Zwängen vermittelt ein positives Gefühl, obwohl oder gerade weil dem Rezipienten natürlich bewusst ist, dass sich dies alles nur auf eine Lebensweltnische, eine zeitlich begrenzte, geschützte Situation bezieht.

Allein das Bewusstsein um dieses „Refugium“ wirkt angenehm, obwohl es zum großen Teil nur eine Option darstellt, die mehr oder weniger genutzt werden kann. Warum das Ausmaß der aktiven Ausnutzung das Unterhaltungserleben beeinflusst, kann durch die Funktion des „kontrollierten Kontrollverlusts“ (Früh 2002: 137) erklärt werden. Er spielt sich auf zwei Ebenen ab. In Bezug auf seine reale Lebenswelt will der Zuschauer keinen Kontroll- und den damit verbundenen Kompetenzverlust riskieren. Durch das permanent aufrecht erhaltene Bewusstsein der Vermitteltheit ist dies gewährleistet, d. h. die unterhaltende Tätigkeit wird als begrenzte und disponible Lebensweltepisode wahrgenommen (Ebene 1). Damit wird es möglich, innerhalb dieser Episode (Ebene 2) mehr oder weniger große Kontrollverluste zu riskieren, um durch die Bestätigung, Wiedererlangung oder Erweiterung der Kompetenz ein positives Erleben zu evozieren. Eine konkrete Variante solcher Prozesse stellt z. B. das Phänomen der Angstlust dar (Vitouch 1993).

Am intensivsten ist das Unterhaltungserleben dann, wenn erstens die Beschäftigung mit dem Gegenstand durch Aufmerksamkeit und Involvement sehr stark in den Vordergrund rückt, komplementär dazu das Bewusstsein der Vermitteltheit stark in den Hintergrund tritt, und wenn zweitens ein möglichst hoher Kontrollverlust auf der Ebene der geschützten Lebensweltepisode (des „Unterhaltungsspiels”; Ebene 2) riskiert und im Vollzug wiedergewonnen wird. Dies wird als Kompetenzbestätigung bzw. Kompetenzgewinn positiv erlebt. Wie sehr sich der Rezipient in Form von Involvement engagieren will, und wie viel Kontrollverlust er damit riskieren möchte, bleibt ihm überlassen (Souveränität).

\subsection{Zusammenfassung}

Es lassen sich somit vier Bedingungen festhalten, welche gegeben sein müssen, um von Unterhaltungserleben zu sprechen:

1. Es muss sich um eine Makroemotion handeln,

2. die zumindest einen kleinen positiven Valenzanteil besitzt;

3. es muss passive Souveränität gegeben sein;

4. es muss aktive Souveränität gegeben sein.

Wenn also das Erleben insgesamt als positiv empfunden wird, keine Zumutungen im Fokus der Aufmerksamkeit liegen und der Rezipient in Gedanken diese Freiräume aktiv ausnutzt, sprechen wir von einem maximalen Unterhaltungserleben. Liegen die genannten Kriterien in graduell abgestufter Form vor (nur etwas positiv, nicht alle Zumutungen vollständig beseitigt, keine vollständige Ausnutzung der Freiräume), dann ist das Unterhaltungserleben entsprechend mehr oder minder stark beeinträchtigt (graduelle Variation). 
Die im Gesamtmodell (Abb. 1) genannten Faktoren verhalten sich zwar zum Teil auch additiv zueinander. Sie stellen aber insbesondere notwendige Bestandteile dar, die das Unterhaltungserleben ganz verhindern, wenn sie nicht wenigstens minimal vorhanden sind. Es handelt sich somit um die konstitutiven Elemente des Konstruktes „Unterhaltungserleben":

1. Liegt überhaupt kein positives Erleben vor, selbst dann nicht, wenn passive und aktive Souveränität gegeben sind, handelt es sich nicht um Unterhaltung. Hier können wir uns z. B. einen Rezipienten vorstellen, welcher aufgrund einer Empfehlung von Freunden oder weil er die Hauptdarstellerin bewundert voller „Unterhaltungserwartung“ ins Kino geht. Dort bestehen für ihn keinerlei „Zumutungen“ (passive Souveränität). Aktive Souveränität in Form von Empathie für die Protagonistin ist infolge seiner positiven Voreinstellung ebenfalls gegeben. Wenn sich bei ihm dann aber trotz dieser „Vorschussinvestition“ einfach kein angenehmes Gefühl einstellen will, wird er sich auch nicht unterhalten haben (z. B. weil ihn „schwarzer Humor überhaupt nicht anspricht, der Film trivial oder langweilig ist o. Ä.).

2. Auch ohne passive Souveränität (also bei Wahrnehmung von „Zumutungen“) handelt es sich trotz ggf. positivem Erleben nicht um Unterhaltung, sondern um eine andere positive Emotion. Stellen wir uns hier z. B. einen Börsenspekulanten vor, welcher in den Nachrichten die neuesten Aktienkurse erfährt und sieht, dass er nunmehr reich ist. Bei ihm wird sich vermutlich gerade wegen der Konsequenzen für den Alltag und der somit fehlenden passiven Souveränität ein angenehmes Gefühl einstellen, was aber eher Erleichterung, Freude, Stolz o. Ä. und nicht Unterhaltung sein wird.

3. Auch bei aktiver Souveränität handelt es sich um eine notwendige Bedingung für Unterhaltung: Je geringer sie ausfällt, also je weniger die durch passive Souveränität geschaffenen Freiräume aktiv genutzt werden, desto geringer fällt das Unterhaltungserleben aus. Ein Rezipient muss sich aber zumindest in geringem Umfang vom Stimulus mitreißen lassen, mitfühlen, mitdenken ${ }^{11}$ oder aber, angestoßen von der erzählten Geschichte, ins Tagträumen geraten, Überlegungen anstellen und Schlussfolgerungen ziehen, wenn wir von Unterhaltung sprechen wollen. Bei gegebenem positiven Erleben und passiver Souveränität sprechen wir also nicht von Unterhaltung, wenn der Rezipient im dargestellten Sinne nicht „aktiv“ wird oder sich seine Aktivität nicht auf den Stimulus bezieht. Die erste Variante lässt sich durch einen jungen Mann illustrieren, der seiner Freundin einen Film auf einer DVD schenken will. Sie mag Fantasyfilme, er überhaupt nicht. Er schaut sich im Laden völlig ungestört (passive Souveränität) einen Film an und ist sofort begeistert, weil er genau den Geschmack seiner Freundin trifft (positive Valenz). Dennoch wird er sich nicht auf den Film „einlassen“ (aktive Souveränität) und damit auch nicht unterhalten, weil Fantasy eben nicht seinen Geschmack trifft. Als Beispiel der zweiten Variante können wir uns eine Person vorstellen, welche an einem schönen Frühlingstag gut gelaunt im Park sitzt (positives affektives Erleben), keinerlei Verpflichtungen hat und in Gedanken frei und gelöst ist (passive Souveränität). Wenn sie sich an der Sonne, dem Vogelgezwitscher oder den vorbeigehenden Damen ergötzt (und dabei z. B. in der Fantasie an ihre Jugend denkt), wird sie sich sicherlich gut unterhalten können. Von der hier relevanten, medienvermittelten Unterhaltung werden wir aber erst dann spre-

11 Ein Rest an Vermitteltheit muss aber immer vorhanden sein, sonst wird z. B. aus „Als-ob-Todesangst“ echte Todesangst (Früh 2002: 132ff.). 
chen können, wenn die angenehmen Fantasien z. B. durch die Lektüre eines mitgebrachten Buches angestoßen werden.

So wie einzelne Kriterien der aktiven Souveränität wenigstens in minimaler Ausprägung vorhanden sein müssen, um Unterhaltung überhaupt zu ermöglichen, so gibt es andere Kriterien, die sich wechselseitig zumindest teilweise substituieren können. Dies ist dann der Fall, wenn die Art der Stimuli ganz bestimmte Kriterien in den Vordergrund stellen, so dass andere eher nebensächlich werden. Wenn ein Krimi sehr spannend gemacht ist, dann spielt es wohl keine große Rolle, dass man dabei nichts lernen kann. Dagegen stellt Spannung bei einem Dokumentarfilm allenfalls ein Zusatzkriterium dar, während der Lerneffekt und ggf. Ästhetik eine dominierende Rolle spielen. In diesem Sinne gibt es eine begrenzte Substituierbarkeit der Unterhaltungskriterien, weil sich der Rezipient immer auf einen dominanten Aspekt konzentriert, aus dem er seine Gratifikationen hauptsächlich bezieht (dominante Rezeptionsstrategie). Zur Erreichung der aktiven Souveränität kann er sich also auf jeweils eine der vier vorgestellten Strategien (kognitiv vs. affektiv, internal vs. external) oder eine bestimmte Kombination (z. B. kognitiv-internal) stützen. Das Ergebnis (ggf. Unterhaltungserleben) ist auf der hier verfolgten Abstraktionsebene das Gleiche. Ein Messinstrument zur Unterhaltung muss deshalb die zentralen und charakteristischen Strategien aller Kontexte (Genres, Situationen) bzw. die dominant unterhaltungsrelevanten Kriterien der Rezipienten zumindest auf hoher Abstraktionsebene selektiv berücksichtigen.

Aus den genannten Unterhaltungskriterien ergeben sich für ein Messinstrument folgende Anforderungen:

- Es muss Unterhaltung als spezifische positive Emotion von anderen positiven Emotionen differenzieren.

- Es muss Unterhaltung in beliebigen Kontexten (z. B. auch in so genannten „nicht unterhaltenden Genres“) identifizieren.

- Es muss Souveränität/Kontrolle in verschiedenen Varianten erfassen.

- Es muss als unabhängiges stetiges Merkmal den Unterhaltungsanteil am Rezeptionserleben graduell abbilden.

Keines der vier Kriterien kann durch die undifferenzierte Frage: „Wie gut haben Sie sich unterhalten?“ erfüllt werden (vgl. auch Schmid \& Wünsch 2001).

\section{Operationalisierung}

\subsection{Datenerhebung}

Die triadisch-dynamische Unterhaltungstheorie legt mindestens zwei verschiedene methodische Abbildungen von Unterhaltungserleben nahe: eine statische und eine dynamische. Wie eingangs erwähnt, wollen wir uns hier nur mit einer statischen Messung beschäftigen, ${ }^{12}$ die postrezeptiv erhoben wird. Sie kann sich auf das momentane Erleben oder retrospektiv auf das Erleben während der gerade abgeschlossenen Rezeption beziehen. Da es uns um eine Vergleichbarkeit zu den traditionell angewandten Erhebungsverfahren geht, beschreiben wir hier zunächst einmal nur die postrezeptive Mes-

12 Zur Berücksichtigung der dynamischen Komponente (vgl. die „dynamische Betrachtung“ in Früh 2002: 140ff.) ist eine rezeptionsbegleitende Messung erforderlich, die von den Autoren ebenfalls eingesetzt wurde. 
sung des Unterhaltungserlebens, die sich retrospektiv auf die Wahrnehmungen während der vorherigen Rezeption bezieht. Die dafür geeignete Datenerhebungsmethode ist die Selbstauskunft der Rezipienten im Rahmen einer Befragung. ${ }^{13}$

Wir schlagen eine getestete Kurzform vor, die ohne großen Aufwand in jeder Survey-Untersuchung einsetzbar ist. Sie ist als Extrakt elaborierterer Messinstrumente entstanden, die in unterschiedlichen empirischen Erhebungen eingesetzt wurden. Die hier nicht beschriebene Langfassung misst Unterhaltung sicherlich genauer als die Kurzform, allerdings um den Preis eines höheren Aufwandes.

Wir benutzen derzeit folgenden einleitenden Fragetext, der je nach Stimulustyp angepasst werden muss: „So, jetzt noch einmal mit Abstand betrachtet: Was empfanden oder fühlten Sie so im Großen und Ganzen beim Anschauen des Beitrags (Films, Sendung, Talkshow etc.)? Beschreiben Sie Ihren Eindruck ganz allgemein, ohne jetzt an bestimmte Einzelheiten zu denken." Durch diese Formulierungen soll sichergestellt werden, dass erstens das eigene Erleben (und nicht der Stimulus) und zweitens die Makroebene des Erlebens (s. o.) angesprochen und vom Befragten beschrieben bzw. bewertet wird (und nicht etwa nur einzelne markante Eindrücke ${ }^{14}$ ).

\section{Valenz}

Zur Erfassung der Valenz wird auf das häufig verwendete Verfahren (vgl. Debus 2000) von Adjektivlisten in Verbindung mit Ratingskalen zurückgegriffen. Positive Valenz lässt sich bei diesem Vorgehen am besten mit dem Adjektiv „angenehm“ beschreiben. Hier sehen wir erstens eine besonders hohe semantische Nähe zu dem zu erfassenden Konstrukt „positive Valenz“, zweitens zeigte sich bei unserer faktorenanalytischen Auswertung mehrerer Studien mit ganz verschiedenen Stimuli, verschiedenen Populationen und teils unterschiedlicher Vorgehensweise, dass dieses Item als einziges jedes Mal hoch auf dem Valenzfaktor lud (und somit eine Art „Universal-Item“ darstellt). Dennoch bleibt eine Unschärfe: Der Ausdruck „angenehm“ ist stark mit einem hedonistischen „Spaß-Aspekt“ (Lust-Unlust-Dimension) konnotiert. Der von Früh verwendete Begriff einer positiven Valenz ist jedoch weiter gefasst. Bei „angenehm“ ist es deshalb denkbar, dass Probanden zumindest in bestimmten Kontexten und anlässlich bestimmter Stimuli die hedonistischen Bedeutungsanteile der Frage „überbewerten“. Dadurch wird die volle Bandbreite der Skala nicht benutzt, weil die eindeutig vorhandene positive Valenz sich teilweise nicht auf hedonistische Spaßgefühle, sondern z. B. auf das schöne Gefühl bezieht, anlässlich eines insgesamt eher unangenehmen Themas etwas gelernt zu haben. Bei der Operationalisierung wird deshalb das Item „angenehm“ mit weiteren Items ergänzt, die eine weniger hedonistische Färbung beinhalten und semantisch

13 Diese Kurzform ist im Anhang komplett als Anwendungsempfehlung abgedruckt.

14 Natürlich muss man annehmen, dass bestimmte Szenen stärker als andere in dieses Urteil eingehen. Z. B. wird hier sicherlich die letzte Szene (z. B. in Form eines Happy Ends) eine besondere Rolle spielen. Solche Effekte lassen sich einerseits als Recency-Effekt durch kognitives Priming (Roskos-Ewoldsen, Roskos-Ewoldsen \& Dillmann Carpentier 2002) beschreiben und würden so eher als Messfehler zu deklarieren sein. Dieser Effekt wird aber gerade in Form der Informations- und Emotionsverabeitungsmodelle (siehe FN 2; Schnotz 1988) von uns theoretisch postuliert. Vor diesem theoretischen Hintergrund wären diese Effekte nicht als Messfehler zu bezeichnen. Zur Klärung dieser Frage muss aber zunächst empirisch geklärt werden, wie stark diese Effekte sind und welche Mechanismen ihnen zugrunde liegen. 
ähnlich universell sind, d. h. auf unterschiedliche Stimuli passen, jedoch besser das nichthedonistische Spektrum positiver Valenz abdecken: „erfreut“, „gut“, „unzufrieden“ und "negativ“. ${ }^{15}$

\section{Passive und aktive Souveränität ${ }^{16}$}

Sowohl bei passiver als auch bei aktiver Souveränität muss die konkrete Operationalisierung deren Subdimensionen "gesellschaftlich-kultureller Kontext“, „Alltagsrelevanz" und „situativer Kontext" bzw. ,internal vs. external“ und „kognitiv vs. affektiv“ (s. o.) getrennt erfassen.

Die drei Dimensionen passiver Souveränität werden über die Zustimmung bzw. Ablehnung zu den folgenden Aussagen über Ratingskalen erfasst:

- gesellschaftlich-kultureller Kontext (keine Konflikte zwischen Stimulus und gesellschaftlich-kulturellen Normen): „Manchmal konnte ich den Film (oder Beitrag, Sendung, Talkshow etc.) nicht mehr richtig genießen, weil mich das Gezeigte abgestoßen hat." 17

- Alltagsrelevanz (Relevanz des Medienbeitrags für den Alltag/über die Rezeption hinaus): „Das Gezeigte ist wichtig für mein normales Leben“18 und „Das Gezeigte betrifft mich persönlich in meinem Alltag." ${ }^{19}$

- situativer Kontext (keine Restriktionen aus dem situativen Kontext: „Ich konnte abschalten und den ganzen Alltag mit Familie, Schule oder Beruf vergessen“ und „Ich hätte eigentlich viel lieber etwas ganz anderes gemacht." 20

Die aktive Souveränität bezeichneten wir als Strategie, mit der ein Medienbeitrag rezipiert wird. Sie ist bestimmt durch Involvement und den Umfang der (selbstbestimmten) externen oder internen Stimulation, die wiederum kognitiver oder emotionaler Art sein kann. $\mathrm{Zu}$ ihrer Messung benutzen wir Adjektive in Verbindung mit Ratingskalen: „nachdenklich“ (kognitiv-internal), „interessiert“ (kognitiv-external), „verträumt“21 (affektiv-internal) und „mitfühlend“ (affektiv-external).

Es liegen nun Messwerte für acht (Unter-)Konstrukte vor:

1. (positive) Valenz

2. passive Souveränität - gesellschaftlich-kultureller Kontext

3. passive Souveränität - Alltagsrelevanz

15 „Unzufrieden“ und „negativ“ sind die negativen Formulierungen von „zufrieden“ und „positiv" und müssen in der Analyse umgepolt werden.

16 Wir erinnern an dieser Stelle noch einmal an den oben formulierten Hinweis, dass wir hier und im Folgenden nur aus stilistischen Gründen „Souveränität/Kontrolle“ als „Souveränität“ abkürzen. Der Inhalt bleibt identisch.

17 Es handelt sich hier um negative Formulierungen, so dass Zustimmung niedrige Werte für passive Souveränität bedeuten.

18 Dito.

19 Dito.

20 Dito.

21 Dieses Adjektiv wird unter Vorbehalt verwendet. Es besitzt u. E. eine etwas zu negative Konnotation und ist zu spezifisch, um die ganze semantische Bandbreite des Konstrukts abzudecken: Fantasievolle Gedankenspiele, Assoziationen signifikanter Erinnerungen (Urlaub, Jugendzeit etc.), kreative Wunschvorstellungen und sonstige affektive Elaborationen. In Ermangelung eines treffenderen Adjektivs bleiben wir jedoch vorerst bei „verträumt“ als beste Annäherung. 
4. passive Souveränität - situativer Kontext

5. aktive Souveränität - internal-affektiv

6. aktive Souveränität - internal-kognitiv

7. aktive Souveränität - external-affektiv

8. aktive Souveränität - external-kognitiv

Dort wo für eine der Subdimensionen mehrere Indikatoren bzw. Aussagen zum Einsatz kommen, werden diese als Likert-Skala interpretiert und die Messwerte (innerhalb einer Subdimension!) arithmetisch gemittelt.

\subsection{Indexbildung}

Da jedes der drei Konstrukte „positive Valenz“, „passive Souveränität“ und „aktive Souveränität“ eine notwendige Bedingung für Unterhaltung darstellt, wird ein multiplikativer Index gebildet, welcher die Intensität bzw. den Umfang des Unterhaltungserlebens angibt:

$$
U I=V \cdot p S \cdot a S
$$

(Unterhaltungsindex $=$ Valenz $*$ passive Souveränität * aktive Souveränität)

Eine empirische Nullausprägung (keine positive Valenz oder keine Souveränität) muss auch als numerische Null kodiert werden. Dieses multiplikative Vorgehen (mit Nullkodierung) weist vier für uns wichtige Merkmale auf.

1. Sobald auch nur eine Inputvariable eine Nullausprägung besitzt, beträgt der gesamte Indexwert Null (negativer Bedingungscharakter der Inputvariablen).

2. Da die Funktion der Indexberechnung einer kubischen Form folgt, werden hohe Indexwerte nur dann erzielt, wenn alle drei Inputvariablen hohe Ausprägungen erreichen. Maximales Unterhaltungserleben wird also nur identifiziert, „wenn alles stimmt" (positiver Bedingungscharakter der Inputvariablen).

3. Der Index identifiziert Unterhaltung auch schon dann, wenn eine oder auch alle Inputvariablen sehr niedrige Werte aufweisen (Unterhaltungserleben als stetiges Merkmal - graduelle Variation).

4. Unterhaltungserleben in unterschiedlichstem Kontext (z. B. verschiedene Genre) wird durch diese multiplikative Indexbildung vergleichbar: Für die Höhe des Unterhaltungsindizes ist es zuerst einmal gleichgültig, ob das Unterhaltungserleben insbesondere aus der interessierten Auseinandersetzung mit einem anspruchsvollen Text oder insbesondere aus der (restriktions-) freien Rezeption einer lustigen TV-Serie herrührt (genreunabhängige Unterhaltungsmessung). ${ }^{22}$

Die drei Unterkonstrukte der passiven Souveränität werden als ungewichteter Mittelwert zum Index „Passive Souveränität“ verrechnet. Dem liegt die Überlegung zugrunde, dass diese drei Dimensionen in den meisten Fällen ${ }^{23}$ miteinander „verrechenbar“ sind: Wir gehen davon aus, dass das Unterhaltungserleben umso stärker beeinträchtigt ist, je mehr Zumutungen auf allen drei Dimensionen insgesamt auftreten und im Umkehrschluss, dass das Unterhaltungserleben mit der „Summe“ der Souveränität auf allen drei Dimensionen steigt. Da die vier Unterkonstrukte der aktiven Souveränität jeweils

22 Dadurch, dass Aggregation (zur Herstellung der Vergleichbarkeit) erfolgt, geht die Information der einzelnen Items über die Tönungen des Unterhaltungserlebens (z. B. bei verschiedenen Genres) nicht verloren, sondern kann bei Bedarf auch einzeln verwendet werden. Dieses Potenzial lässt sich in einer Langfassung des Messinstrumentes weiter spezifizieren.

23 Es handelt sich hierbei um eine operationale Vereinfachung. 
unterschiedliche Rezeptionsstrategien darstellen (s. o. 1.2 Zusammenfassung, Abschnitt 3), muss hier ein anderes Verfahren der Indexbildung gewählt werden. Der Index für aktive Souveränität wird deshalb lediglich aus dem Messwert für dasjenige Unterkonstrukt (bzw. die Rezeptionsstrategie) gebildet, welches die höchste Ausprägung besitzt.

Valenz weist keine Unterkonstrukte auf, so dass wir hier einfach mit dem Mittelwert der fünf vorgestellten Valenzindikatoren arbeiten. Aus diesen Überlegungen entsteht folgende Formel: ${ }^{24}$

$$
U I=V \cdot\left(\frac{p S_{g k K}+p S_{A R}+p S_{s K}}{3}\right) \cdot \max \left\{a S_{i a} ; a S_{i k} ; a S_{e a} ; a S_{e k}\right\}
$$

Da dieser Wert skalenabhängig ist, ${ }^{25}$ schlagen wir einen standardisierten Index mit einem Wertebereich von 0 bis 100 nach folgender Berechnungsvorschrift vor:

$$
U I_{100}=U I \cdot \frac{100}{a \cdot b \cdot c}
$$

$\mathrm{a}=$ (theoretischer) Skalenendpunkt Valenz

$\mathrm{b}=$ (theoretischer) Skalenendpunkt passive Souveränität

$\mathrm{c}=$ (theoretischer) Skalenendpunkt aktive Souveränität

\section{Validierung}

\subsection{Beweislogik und Hypothesen}

Die Güte des Messinstrumentes lässt sich anhand von Reliabilität und Validität beschreiben. Zur Prüfung dieser Kriterien werden verschiedene empirische Verfahren vorgeschlagen (vgl.: Bortz, Döring 2002: 193ff.; Schnell, Hill, Esser 1999: 143ff. und Grubitzsch 1999: 146ff.).

a) Reliabilität: Reliabilitätstests fordern entweder eine zeitliche Stabilität der Messung (bei unverändertem Gegenstand) oder beruhen auf der Annahme der Homogenität äquivalenter Messungen. Beide Strategien sind in unserem Fall nur bedingt anwendbar: Die Bedingung des unveränderten Gegenstandes ist hier auf der Individualebene bereits aufgrund unserer theoretischen Argumentation nicht erfüllbar, weil der Rezipient mit der Messung transagiert. Er wird sich mit demselben Stimulus nicht zweimal auf die gleiche Art und Weise unterhalten. Denn dadurch, dass der Inhalt bei der zweiten Rezeption bekannt(er) ist, handelt es sich zwangsläufig um einen „anderen“ Stimulus. Auch

$24 \mathrm{UI}=$ Unterhaltungsindex; $\mathrm{V}=$ positive Valenz; pSgkK = passive Souveränität - gesellschaftlichkultureller Kontext; pSAR = passive Souveränität - Alltagsrelevanz; pSsK = passive Souveränität - situativer Kontext; aSia = aktive Souveränität - internal, affektiv; aSik = aktive Souveränität - internal, kognitiv; aSea = aktive Souveränität - external, affektiv; aSek = aktive Souveränität - external, kognitiv.

25 Wurden z. B. die Unterkonstrukte alle auf einer 7-stufigen Skala erhoben, ergibt sich ein Wertebereich von 0 bis 216 (da gefordert wird, dass das untere Ende jeder berücksichtigten Skala mit 0 kodiert wird, haben 7-stufige Skalen eine Range von 0 bis 6. Der Maximalwert für den Unterhaltungsindex berechnet sich somit als $6 \cdot 6 \cdot 6=216$ ). Wurden die Konstrukte auf einer 5 -stufigen Skala erhoben, ergibt sich ein Wertebereich von 0 bis 64 (Der Maximalwert für den Unterhaltungsindex ergibt sich hier analog als $4 \cdot 4 \cdot 4=64$ ). 
wenn sich deshalb die zeitliche Stabilität des Messinstruments mit einer Test-Retest-Anordnung auf Individualebene nicht prüfen lässt, so können wir doch davon ausgehen, dass zumindest auf einer Aggregatebene, also durch Mittelung vieler unterschiedlicher Individuen und situativer Kontexte, die Ergebnisse durch Gruppenvergleich replizierbar sein müssen. ${ }^{26}$ Dies prüfen wir mit folgender Hypothese:

$H_{1}$ : Ein gleicher andiovisueller Stimulus fübrt anf der Aggregatebene bei verschiedenen statistisch vergleichbaren Personengruppen in gleichen Kontexten zu gleichen Werten für das Unterhaltungserleben.

Konkret heißt $\mathrm{H}_{1}$ also: Bei ein und demselben Stimulus in zwei verschiedenen Untersuchungen unterhält sich Population 1 im Durchschnitt genauso wie eine vergleichbare Population 2.

Reliabilitätsprüfungen, welche auf der Annahme der Homogenität äquivalenter Messungen beruhen, setzen natürlich mehrere äquivalente Messungen (üblicherweise in Form von verschiedenen Items aus einem größerem Pool) voraus. Insbesondere im Hinblick auf unser Ziel, das Messinstrument in der Survey-Forschung ebenfalls effizient anwenden zu können, ist es jedoch kaum möglich und sinnvoll, große Itempools zu verwenden. Dadurch sind diese Gütetests hier allenfalls bedingt ${ }^{27}$ anwendbar und werden deshalb nicht durchgeführt. ${ }^{28}$

b) Validität: Hier sind für uns Face- und Konstruktvalidität anwendbar. Facevalidität beschreibt die „augenscheinliche“ Validität eines Messinstrumentes. Eine systematische Prüfung kann hier als eine Plausibilitätsprüfung, z. B. in Form eines Gedankenexperiments erfolgen. $\mathrm{Zu}$ prüfen ist dabei, ob das Messinstrument auf eine extreme, systematische Variation der konstituierenden Bedingungen theoriegemäß reagiert und dabei die Skalenendpunkte auch erreicht. Eine solche Prüfung führten wir für das Vorgehen bei der Indexberechnung (unter 2.2) durch und gelangten zu positiven Ergebnissen (vgl. dazu auch die Minima und Maxima in Abb. 2 als empirische Bestätigung). Andere Aspekte der Facevalidität beschränkten sich auf die Prüfung, ob bestimmte Konstruktionsprinzipien unserer Formel nicht doch zu Situationen führen können, für die überhaupt keine plausiblen konkreten „Fälle“ mehr vorstellbar sind. Beispiel: Wenn die passive Souveränität gleich null ist, wird gemäß der Formel auch das Unterhaltungserleben null. Lassen sich nun plausible (extreme) Situationen vorstellen, in denen dies nicht der Fall ist? Auch diese „Rückübersetzung“ der Formelkonstruktion führte zu keinen fehlerhaften (d. h. hier unplausiblen) Resultaten. Durch diese positive Prüfung wird außerdem das oben genannte Kriterium erfüllt, dass ein positives Unterhaltungserleben von anderen positiven Emotionen unterscheidbar wird.

Konstruktvalidität liegt vor, wenn sich aus dem vorliegenden Konstrukt Hypothesen über Beziehungen zu anderen Konstrukten herleiten und empirisch bestätigen

26 Führen diese Untersuchungen außerdem verschiedene Untersuchungsleiter durch (wie hier geschehen) und die Hypothesen werden bestätigt, ist ebenfalls davon auszugehen, dass das Messinstrument vom Anwender unabhängig („objektiv“) misst.

27 Cronbachs alpha als der bekannteste Homogenitätskoeffizient „unterschätzt“ die Reliabilität bei einer geringen Itemanzahl, so dass z. B. bei einer Batterie von zwei Items mit perfekter Interitemkorrelation nur ein alpha von 0,5 und bei einer Batterie von drei Items von nur 0,66 resultiert.

28 Dennoch wurde die Homogenität in Form von einfachen Interitemkorrelationen - soweit möglich - von uns geprüft. Wir konnten überall zufrieden stellende mittlere $(>0.3)$ bis starke Korrelationen feststellen. 
lassen. Dieses Kriterium ist für unser Messinstrument gut prüfbar, da bereits die theoretische Herleitung des Konstruktes „Unterhaltungserleben“ (Kap.1 und Früh 2002) zahlreiche Grundlagen zur Formulierung empirisch prüfbarer Hypothesen bereitstellt.

$\mathrm{H}_{2}$ : Inbaltlich und/oder formal unterschiedliche audiovisuelle Stimuli fübren zu unterschiedlichen Werten für das Unterhaltungserleben.

Der rezipierte Inhalt stellt eine zentrale Einflussgröße dar (siehe triadisches Ursachenfitting; Früh 2002). Ändert er sich (bei Konstanz der anderen Größen auf Aggregatebene), dann ändert sich auch das Unterhaltungserleben. ${ }^{29}$

\section{$\mathrm{H}_{3}$ : Unterschiedliche situative Kontexte der Rezeption fübren zu unterschiedlichen Werten für das Unterhaltungserleben.}

Ändert sich das situative Umfeld der Rezeption (bei Konstanthaltung der anderen Einflussgrößen auf Aggregatebene), muss sich auch das Unterhaltungserleben ändern (siehe triadisches Ursachenfitting; Früh 2002).

\section{$\mathrm{H}_{4}$ : Je besser der Stimulus den Präferenzen der Rezipienten entspricht, desto mehr unterhalten sie sich.}

Die nach dem triadischen Modell dritte Einflussgröße stellt die Person mit ihren relevanten Merkmalen dar. Allerdings lassen sich hierbei, von wenigen Ausnahmen abgesehen, wie z. B. Reflexreaktionen, keine theoretisch plausibel begründeten Merkmale finden, welche allein und generell ein unterschiedliches Unterhaltungserleben herbeiführen. Es ist nicht anzunehmen, dass sich z. B. Männer generell besser als Frauen oder alte generell besser als junge Menschen unterhalten. ${ }^{30}$ Personelle Merkmale werden hier erst „unterhaltungswirksam“, wenn man sie in Kombination (Transaktion) mit weiteren Elementen der Triade betrachtet. Personen, welche z. B. „anspruchsvolles Programmkino" mögen, werden sich nicht generell besser unterhalten, sondern nur dann, wenn sie Filme sehen, welche diesen Ansprüchen, Vorstellungen und Präferenzen genügen.

\section{$H_{5:}$ Es besteht nur ein geringer bis mäßig starker Zusammenhang zwischen dem Unterhaltungserleben und der Benennung als Unterbaltung durch die Rezipien- ten.}

Ein weiterer, bisher nicht berücksichtigter Aspekt der Konstruktvalidität stellt die diskriminante Validität dar. Geprüft wird, ob das Messinstrument tatsächlich etwas anderes misst als die Operationalisierungen anderer, ähnlicher Konstrukte. Dies ist insbesondere dann zu prüfen, wenn aufgrund der inhaltlichen Verwandtschaft zweier ähnlicher Konstrukte die Gefahr besteht, dass die Operationalisierungen der Konstrukte diese Differenzen nicht mehr abbilden können.

29 Wobei natürlich nicht ausgeschlossen ist, dass es individuelle Fälle gibt, bei denen das Unterhaltungserleben bei zwei sehr verschiedenen Stimuli (nahezu) identisch ist. Diese Annahme ist auch völlig theoriekonform, berührt aber diese auf Aggregatebene argumentierende Hypothese nicht.

30 Diese Argumentation gilt ebenfalls nur auf „aggregierter Ebene“, d. h. es gibt sehr wohl bestimmte Filme oder Situationen, in denen sich Frauen besser als Männer oder Junge besser als Alte unterhalten. Diese Unterschiede verschwinden aber im Hinblick auf alle möglichen Stimuli oder Situationen. So zeigte sich z. B. in unseren Daten kein signifikanter Unterschied im Grad des Unterhaltungserlebens zwischen Männern und Frauen. Lediglich beim Spielfilm „The Cell“ mit der Hauptdarstellerin Jennifer Lopez haben sich Männer signifikant mehr unterhalten als Frauen. Es sei nun dahingestellt, ob man dies auf die Vorliebe von Männern für dieses Genre zurückführen will oder auf eine Affinität für die Hauptdarstellerin. 
Früh argumentiert, dass das operational verwendete Label „unterhaltend“ zwar auch Unterhaltungserleben im hier verwendeten Sinne erfasst, es wird aber zusätzlich durch sozialisierte Genrevorstellungen und Begriffskonnotationen überlagert (Früh 2002: 76ff.). Dieses traditionell erhobene Konstrukt ist somit unserem zwar sehr ähnlich, stellt letztlich aber dennoch ein anderes Konstrukt dar. Die Operationalisierungen beider Konstrukte sollten also neben Übereinstimmungen auch $\mathrm{zu}$ differenten Ergebnissen führen. Diese Differenz sollte besonders stark bei Medienangeboten sein, bei denen man auf Grund sozialisierter Genrelabels eher nicht von Unterhaltung spricht, wie in ernsten (z. B. Dokumentationen) oder realen (z. B. Nachrichten) Kontexten. Durch die Art der ausgewählten Stimuli kann damit gleichzeitig geprüft werden, ob unser Messinstrument in der Lage ist, Unterhaltungsanteile auch in nicht dominant unterhaltenden Genres zu erfassen.

\subsection{Validierungsstudien}

Zur Überprüfung dieser Hypothesen und somit zur Validierung unseres Messinstrumentes greifen wir auf die Ergebnisse dreier von uns durchgeführten Studien zurück. Diese wurden im Anschluss an die Formulierung der TDU durchgeführt und hatten einerseits das Ziel, einzelne elementare Grundaussagen der TDU empirisch zu überprüfen und andererseits ein Messverfahren zu entwickeln, das all diese theoretischen Essentials erfasst. ${ }^{31}$ Dabei wurden in der ersten Studie längere und kürzere semantische Differenziale sowie Statementbatterien eingesetzt. In jeder folgenden Studie wurden diese Instrumente optimiert, indem Adjektive ausgetauscht, weitere Statements hinzugefügt oder geringfügig umformuliert wurden etc. Alle Studien waren also Validierungsstudien zur TDU mit lediglich unterschiedlichen Zielsetzungen. Daraus wurde nun als Extrakt eine Kurzform ${ }^{32}$ des Messinstruments entwickelt. Alle darin enthaltenen Aspekte waren auch in jeder Validierungsstudie enthalten. Der einzige systematische Unterschied besteht darin, dass die einzelnen Skalen nicht in dieser Reihenfolge benutzt wurden, da sie jeweils unter andere untergemischt waren. Nach den ersten beiden Studien erfolgte außerdem ein Umstieg von semantischen Differentialen auf die hier vorgeschlagenen Adjektivchecklisten, indem einfach der jeweilige semantische Gegenpol weggelassen wurde. ${ }^{33}$ Wir gehen davon aus, dass sich diese Änderungen auf unseren aus vielen Items zusammengesetzten Indexwert nicht bedeutend auswirken.

\subsubsection{Studie 1: Unterbaltung im nicht unterbaltenden Kontext („Ernste Unterbaltung“)}

Als Stimulus diente in dieser Studie (Klopp 2003) der Dokumentarfilm „Herr Zwilling und Frau Zuckermann“. Es handelt sich dabei um ein preisgekröntes Porträt deutschsprachiger Juden, die im jetzt ukrainischen Czernowitz den Holocaust überlebten. Der

31 Es wurde also z. B. überprüft, ob die TDU tatsächlich auch Unterhaltung in so genannten „nicht unterhaltenden Genres“ erfasst, ob man eine Mikro- von einer Makroebene differenzieren kann, ob die Messung unabhängig von speziell vorbereiteten Laborsituationen problemlos möglich ist und nicht zu stark unterscheidet etc. (s. u.)

32 Eine Langform existiert als fertiges Instrument nicht, sondern mehrere weitgehend ähnliche Langformen, die auf unterschiedliche inhaltliche Zielsetzungen ausgelegt sind.

33 Dies erwies sich als notwendig, da es bei einigen Items nicht möglich ist, einen semantisch eindeutigen Gegenpol zu formulieren. 
Film enthielt überwiegend traurige Szenen, wie die Beschreibung des Holocausts und das fast zufällige Überleben der Protagonisten, aber auch einige Szenen, welche den Lebensmut, die Neugier und den melancholischen Humor der teils über 80 Jahre alten Hauptpersonen zeigen. In einer Filmversion (Dauer ca. 15 Minuten) wurden nur ernste Szenen zusammengeschnitten, in einer zweiten Version (ca. 20 Minuten) nur (eher) humorvolle Abschnitte, und in einer dritten Version (ca. 35 Minuten) wurden diese ernsten und heiteren Szenen gemischt. Befragt wurden (im Zeitraum von Juli 2002 bis Januar 2003) pro Version ca. 40 Personen, wobei ihnen der Film zu Hause in Anwesenheit des Interviewers vorgespielt wurde. Danach war ein Fragebogen auszufüllen. Die Probanden wurden nach Quotenvorgaben rekrutiert, die Gruppen sind dabei hinsichtlich Alter (Mittelwert 38 Jahre), Geschlecht (51 \% weiblich) und Bildung (62\% Hochschulabschluss oder -reife) egalisiert.

\subsubsection{Studie 2: Unterhaltung unter alltagsnaben Bedingungen („Alltagsunterbaltung “)}

Diese Studie wurde mit dem Ziel der (Konstrukt-)Validierung des Messinstrumentes entwickelt. Die Erhebung fand im Oktober 2002 statt. Es sollte das alltagsnahe Unterhaltungserleben bei zwei Genres verglichen werden: Nachrichten und Spielfilmen. 53 Personen wurden in einer schriftlichen Instruktion gebeten, direkt „nachdem sie das nächste Mal eine Nachrichtensendung oder einen Spielfilm ${ }^{35}$ gesehen haben“, einen Fragebogen zur Erfassung des Unterhaltungserlebens auszufüllen. Dies sollte jeweils für zwei Nachrichtensendungen und zwei Spielfilme geschehen, so dass insgesamt $207^{36}$ Messungen vorliegen. Die (weitest gehende) Alltagsnähe wurde realisiert, indem die Probanden erstens zu Hause in ihrer natürlichen Alltagsumgebung befragt wurden und zweitens die Selektion und Rezeption völlig selbstbestimmt (natürlich im Rahmen der jeweiligen alltäglichen sozialen Situation) war. Die Untersuchungsteilnehmer konnten selber entscheiden, wann sie welche Sendungen anschauten. Es handelte sich um eine Quotenstichprobe mit begrenzten Rekrutierungsmöglichkeiten aus dem Umfeld der Interviewer. Das Durchschnittsalter liegt bei 42 Jahren, Frauen sind leicht überrepräsentiert (63\%), und knapp die Hälfte der Probanden besitzt einen Hochschulabschluss oder die Hochschulreife (44\%). ${ }^{37}$

\subsubsection{Studie 3: Unterbaltung als Wabrnebmungs- und Verarbeitungsprozess („Unterhal- tungsprozess")}

Gegenstand dieser Studie waren unterhaltsame Wahrnehmungs- und Verarbeitungsprozesse während der Rezeption, welche u. a. mittels „Continuous Response Measurement" erfasst wurden (zu CRM siehe auch Biocca/David/West 1994). In Einzeluntersuchungen wurden 289 Probanden im Labor vier Filme vorgeführt (jeden Film sahen also reichlich 70 Personen). Neben der rezeptionsbegleitenden CRM-Messung wurden direkt nach der Rezeption das Unterhaltungserleben erfasst sowie Genrepräferenzen

34 Diese Studie wurde von Kathleen Arendt und Heike Masurek in Zusammenarbeit mit den Autoren durchgeführt.

35 Die beiden Genres wurden den Probanden genauer erläutert.

36 Fünf Personen bearbeiteten den Fragebogen nur nach drei Sendungen.

37 Diese soziodemografischen Daten wurden in einer separaten schriftlichen Befragung erhoben. 
und verschiedene Persönlichkeitsmerkmale erhoben. Alle Filme waren auf eine Länge von ca. 30 bis 40 Minuten geschnitten. Dabei handelte es sich um den Science-FictionHorror-Thriller ${ }^{38}$ „The Cell“ (USA 2000) mit Jennifer Lopez, die Action-Komödie „Grosse Pointe Blank“ (USA 1997), den Drama-Thriller „Funny Games“ (Österreich 1997) sowie den Dokumentarfilm „Herr Zwilling und Frau Zuckermann“ (Deutschland 1999). ${ }^{39}$ Bei diesem Dokumentarfilm handelt es sich um die dritte und längste Version der Studie „ernste Unterhaltung“ (s. o).

Die Datenerhebung fand zwischen Juli 2003 und März 2004 statt. Es handelt sich um eine Quotenstichprobe, welche überwiegend aus dem Umfeld der Interviewer rekrutiert wurde. Das Durchschnittsalter lag bei 29 Jahren, das Bildungsniveau war überdurchschnittlich hoch (83 \% mit Hochschulreife; davon über die Hälfte Studierende) und die Geschlechter waren gleichmäßig verteilt (53\% weiblich). Zwischen den vier Filmgruppen bestanden hinsichtlich dieser Merkmale keine signifikanten Unterschiede.

\subsection{Ergebnisse}

Zur ersten Orientierung sind in Abbildung 2 die ermittelten Unterhaltungswerte aller drei Studien dargestellt. Tabelle 1 enthält außerdem eine Übersicht über die Unterhaltungswerte bei verschiedenen Genres, ${ }^{40}$ die von den Probanden in der Studie „Alltagsunterhaltung“ gesehen wurden. Für unser vorgeschlagenes Vorgehen spricht hier (vgl. Facevalidität), dass bei fünf von neun Settings (Abb. 2) ein individueller Minimalwert von Null erreicht wird und bei drei Settings ein individueller Maximalwert oberhalb von 90 erfasst wurde. Somit werden auch die Extremwerte der Skala empirisch erreicht.

Tabelle 1: Unterhaltungswerte verschiedener Genres und Sendungen der Studie „Alltagsunterhaltung"

\begin{tabular}{|c|c|c|c|c|c|}
\hline Sendung/Genre & $\mathbf{N}$ & $\mathrm{UI}_{100}$ & $\mathrm{SD}$ & Min & Max \\
\hline Nachrichten & 105 & 22,8 & 11,7 & 1,6 & 52,9 \\
\hline Spielfilme allgemein & 102 & 41,7 & 17,6 & 1,59 & 91,8 \\
\hline $\begin{array}{l}\text { Komödien, Slapstick, } \\
\text { Humorsendungen }\end{array}$ & 45 & 47,9 & 17,6 & 7,3 & 91,8 \\
\hline Liebe/Romantik & 29 & 46,2 & 19,2 & 7,3 & 91,8 \\
\hline $\begin{array}{l}\text { Science-Fiction, Fantasy, } \\
\text { Grusel, Horror }\end{array}$ & 17 & 40,2 & 18,0 & 6,6 & 70,6 \\
\hline $\begin{array}{l}\text { melodramatische Filme, } \\
\text { Tragödien }\end{array}$ & 31 & 40,0 & 14,6 & 6,6 & 69,1 \\
\hline $\begin{array}{l}\text { Krimis, Actionfilme, } \\
\text { Agentenfilme }\end{array}$ & 34 & 36,1 & 16,4 & 1,5 & 69,4 \\
\hline
\end{tabular}

38 Die Genrelabels wurden den Internet-Filmdatenbanken http://www.imdb.com und http:// www.ofdb.de entnommen.

39 An dieser Stelle kann der Inhalt bzw. die Handlung der einzelnen Filme nicht vorgestellt werden. Weitere Informationen dazu sind in den genannten Filmdatenbanken zu finden

40 Die Genrezuordnungen wurden im Nachhinein kodiert. Dies geschah unter Rückgriff auf die Genreeinordnung der Internet-Filmdatenbanken www.ofdb.de und www.imdb.com. 


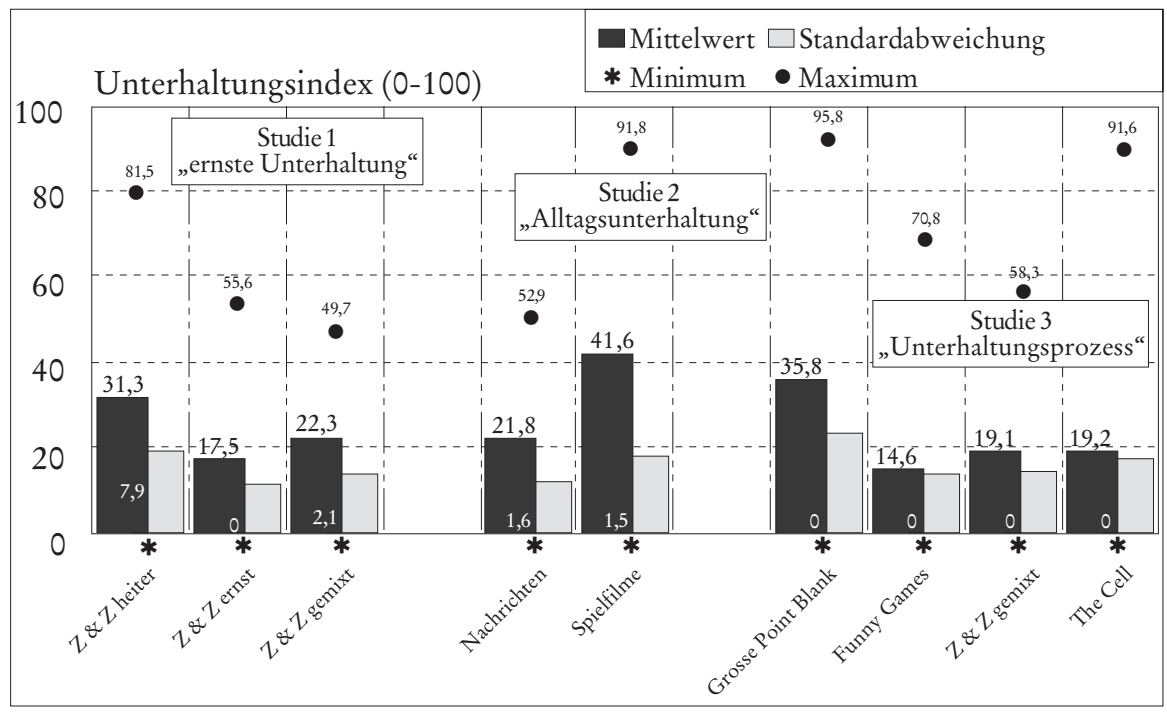

$H_{1}$ : Ein gleicher andiovisueller Stimulus führt auf der Aggregatebene bei statistisch vergleichbaren Personengruppen in gleichen Kontexten zu gleichen Werten für das Unterhaltungserleben.

Der Dokumentarfilm „Herr Zwilling und Frau Zuckermann“ wurde in der 35-minütigen Version (ernste und heitere Elemente) sowohl einer Gruppe der Studie „ernste Unterhaltung“ ( $\mathrm{n}=39)$ als auch einer Gruppe ( $\mathrm{n}=71)$ der Studie „Unterhaltungsprozess“ vorgeführt und anschließend das Unterhaltungserleben erfasst. Die Gruppen waren allerdings aufgrund der Art der Stichproben nicht egalisiert - sie unterschieden sich bezüglich Bildungsstand und Alter (siehe Beschreibung). Da aber beide Merkmale keinerlei ${ }^{41}$ signifikanten ${ }^{42}$ Einfluss auf das Unterhaltungserleben haben, gehen wir davon aus, dass es sich bei den beiden Gruppen für unsere Fragestellung um „statistisch vergleichbare" Gruppen handelt. Das situative Umfeld beider Studien unterschied sich nur gering. ${ }^{43}$ In der ersten Studie führte der Dokumentarfilm im Durchschnitt zu einem Unterhaltungserleben von 22,3 Punkten (auf einer 0-100-Skala) $(\mathrm{SD}=13,5)$, in der dritten Studie "Unterhaltungsprozess" unterhielten sich die Probanden nur geringfügig weni-

41 Ein solcher Zusammenhang konnte weder bei diesem noch bei anderen Filmen oder Studien gefunden werden.

42 Getestet wurde auf einem 5-prozentigen Niveau (2-seitig) mit Pearson-Korrelation bzw. ChiQuadrat.

43 Dabei war die Situation in Studie 1 aufgrund der rezeptionsbegleitenden Messung (die VPN bedienten einen umfunktionierten Joystick als eindimensionalen Schieberegler) geringfügig künstlicher und somit auch vermutlich etwas unterhaltungsmindernder. Dies kann bei der Interpretation der Ergebnisse berücksichtigt werden. 
ger $\left(\mathrm{UI}_{100}=19,1 ; \mathrm{SD}=14,3\right)$ (siehe auch Abbildung 2). Dieser geringe Unterschied ist nicht signifikant $(\mathrm{t}(108)=1,13$; n.s.). Soweit kann also Hypothese 1 als bestätigt gelten und wir können von einer stabilen (reliablen) und personenunabhängigen (objektiven) Messung von Unterhaltungserleben ausgehen.

\section{$\mathrm{H}_{2}$ : Inhaltlich und/oder formal unterschiedliche audiovisuelle Stimuli fübren zu unterschiedlichen Werten für das Unterhaltungserleben.}

Dass unterschiedliche Filme tatsächlich zu unterschiedlichen Unterhaltungswerten führten, zeichnet sich auch schon in Abbildung $2 \mathrm{ab}$. In Studie 1 („ernste Unterhaltung“) hat der Stimulus bei einer Varianzaufklärung von 13 Prozent (Eta) einen signifikanten Einfluss auf die Höhe des Unterhaltungserlebens $(\mathrm{F}(2,117)=7,4 ; \mathrm{p}<.001)$. Dabei unterscheidet sich die „nur heitere“ Version $\left(\mathrm{UI}_{100}=31,3 ; \mathrm{SD}=19,0\right)$ von der „nur ernsten“ Version $\left(\mathrm{UI}_{100}=17,5 ; \mathrm{SD}=11,1\right)$ hochsignifikant. ${ }^{44} \mathrm{Die}$ „Mixversion“ liegt mit einem Unterhaltungsindex von 22,3 (SD=13,5) zwar über dem Wert von Version 2, der Unterschied ist aber nicht signifikant.

Bei Studie 2 „Alltagsunterhaltung“ unterscheiden sich Nachrichten mit einem durchschnittlichen Unterhaltungsindex von 21,8 ( $\mathrm{SD}=11,7)$ hochsignifikant $(\mathrm{t}(205)=9,6$; $\mathrm{p}<.001)$ von den Spielfilmen mit einem durchschnittlichen Unterhaltungsindex von 41,6 $(\mathrm{SD}=17,6)$. Auch bei einer deskriptiven Betrachtung auf der Ebene einzelner Sendungen sprechen die Ergebnisse für unsere Hypothese: ${ }^{45}$ Individueller „Spitzenreiter“ mit einem maximalen Unterhaltungswert von 91,8 war die Liebeskomödie „Verliebt auf Bermuda“. Im Schnitt ${ }^{46}$ wurde die Komödie „Rennschwein Rudi Rüssel“ mit einem Unterhaltungsindex von 58,1 (SD=9,3) am höchsten bewertet. Aber auch Dramen wie z. B. "The Green Mile“ $\left(\mathrm{UI}_{100}=44,4 ; \mathrm{SD}=16,7\right)$ oder „Zu nah am Feuer" $\left(\mathrm{UI}_{100}=38,7\right.$; $\mathrm{SD}=9,2)$ und Thriller wie „Fahr zur Hölle, Schwester" $\left(\mathrm{UI}_{100}=38,3 ; \mathrm{SD}=19,5\right)$ oder „James Bond“ $\left(\mathrm{UI}_{100}=30,0 ; \mathrm{SD}=14,7\right)$ bieten höhere Unterhaltungswerte als die Nachrichtensendungen. ${ }^{47}$ Bei diesen lag das „heute journal“ mit einem Unterhaltungsindex von $24,7(\mathrm{SD}=10,1)$ an der Spitze, dicht gefolgt von „RTL aktuell“ $\left(\mathrm{UI}_{100}=24,2 ; \mathrm{SD}=13,5\right)$. Die wenigsten Unterhaltungsanteile enthielten "mdr aktuell“ $\left(\mathrm{UI}_{100}=17,7 ; \mathrm{SD}=13,7\right)$ und „heute“ $\left(\mathrm{UI}_{100}=16,6 ; \mathrm{SD}=8,8\right)$.

Bei einer Varianzaufklärung von 18 Prozent (Eta) hat der Stimulus auch in Studie 3 („Unterhaltungsprozess“) einen hochsignifikanten $(\mathrm{F}(3,285)=21,05 ; \mathrm{p}<.001)$ Einfluss auf das Unterhaltungserleben. Erwartungsgemäß erreichte die Action-Komödie „Grosse Point Blank“ mit 35,8 (SD=23,4) den höchsten Wert (der Unterschied zu den anderen Filmen ist jeweils hochsignifikant ${ }^{48}$ ). Den niedrigsten Wert erhielt mit 14,6 $(\mathrm{SD}=13,7)$ der Drama-Thriller „Funny Games“. „The Cell“ liegt bei 19,2 $(\mathrm{SD}=17,5)$ und „Herr Zwilling und Frau Zuckermann“ bei 19,1 (SD=14,3). Außer zu „Grosse Point Blank“ ergaben sich aber keine signifikanten Unterschiede zwischen den anderen Filmen.

Es lässt sich also zeigen, dass unser Messinstrument zwischen verschiedenen Stimuli zu differenzieren vermag, wobei die Abweichungen stets in der erwarteten und plausi-

44 Tamhane-T2-post-hoc-Test; $\mathrm{p}=0.001$.

$45 \mathrm{Da}$ die Fallzahlen für einzelne Sendungen z. T. sehr gering sind, sind inferenzstatistische Analysen hier nicht möglich.

46 Im Folgenden werden nur die Ergebnisse von Sendungen dargestellt, die von mindestens drei Personen gesehen wurden.

47 Alle(!) Spielfilme liegen im Schnitt höher als die Nachrichtensendungen.

48 Tamhane-T2-post-hoc-Test; $\mathrm{p}<0.001$. 
blen Richtung lagen: Spielfilme bieten ein größeres Unterhaltungserleben als Nachrichten (Studie 2), die „heitere“ Version eines ernsten Dokumentarfilms ein höheres Unterhaltungserleben als die ernste Version (Studie 1) und eine Action-Komödie ein höheres Unterhaltungserleben als ein Drama oder ein Dokumentarfilm (Studie 3). Hypothese 2 kann also als bestätigt gelten.

\section{$H_{3}$ : Unterschiedliche situative Kontexte der Rezeption führen zu unterschiedlichen Werten für das Unterbaltungserleben.}

Da die drei Studien nicht direkt zu Validierungszwecken für das hier vorgeschlagene Messinstrument konzipiert waren, lassen sich in unserem Material bei identischem Stimulus keine hinreichend unterschiedlichen situativen Kontexte vergleichen. Deshalb müssen wir die Beweisführung von der Beitrags- auf die Genreebene verschieben. Es soll ein globaler Vergleich zwischen den Spielfilmen, die alltagsnah und freiwillig in der „Alltags-Studie" rezipiert wurden, und jenen, die in der Studie "Unterhaltungsprozess" weitgehend unter Laborbedingungen untersucht wurden, ${ }^{49}$ gezogen werden. Es handelt sich also um eine Prüfung auf Genre-, nicht auf Beitragsebene. Der Mittelwert für das Unterhaltungserleben bei Spielfilmen beträgt in der alltagsnahen Studie (Studie 2) 41,7 $(\mathrm{SD}=17,6)$, in der Laborstudie (Studie 3) 23,3 $(\mathrm{SD}=20,7)$. Der Unterschied ist dabei hochsignifikant $(\mathrm{t}(318)=7,7 ; \mathrm{p}<0.001)$. Hypothese 3 kann also unter Berücksichtigung der gegebenen Einschränkungen als bestätigt gelten. ${ }^{50}$

\section{$H_{4}:$ Je stärker der Film den Präferenzen der Rezipienten entspricht, desto mebr un- terhalten sie sich.}

Die Probanden wurden in der Studie „Unterhaltungsprozess“ gebeten anzugeben, welche Merkmale (ihre Lieblings-)Sendungen im Fernsehen haben müssen, damit sie ihnen gut gefallen..$^{51}$ Mit einer Batterie von 17 Aussagen ${ }^{52}$ wurden so ihre inhaltlichen und formalen Präferenzen auf sechs verschiedenen Merkmalsdimensionen erhoben: 1) subjektive Nähe, Realismus und Relevanz des Inhaltes, 2) inhaltliche und formale Dynamik des Dargestellten, 3) handwerkliche, ästhetische, künstlerische, thematische Qualität, 4) humorvolle und/oder kuriose Inhalte oder entsprechende Darstellung, 5) Darstellung oder Thematisierung unangenehmer, negativ besetzter Themen oder Evokation negativer Emotionen, 6) Darstellung oder Thematisierung angenehmer, positiver Themen oder Evokation positiver Emotionen.

Die verwendeten vier Filme wurden von uns auf diesen Dimensionen eingestuft. Wir codierten nur jene Merkmalsdimensionen, auf denen der jeweilige Stimulus stark überoder unterdurchschnittliche Ausprägungen aufwies. So wird man z. B. dem DramaThriller „Funny Games“ durchaus künstlerische, handwerkliche und auch ästhetische Qualitäten zusprechen, und er enthält auch einige komische und humorvolle Szenen.

49 Also die Filme „Grosse Point Blank“, „Funny Games“ und „The Cell“.

50 Auch hier gilt, dass die beiden verglichenen Gruppen nicht egalisiert waren. Da aber die soziodemographischen Merkmale keinen Einfluss auf das Unterhaltungserleben haben (siehe auch Anmerkungen zu Hypothese 1), gehen wir für unsere Fragestellung von vergleichbaren Gruppen aus.

51 „Wenn Sie jetzt bitte noch einmal an all die Sendungen im Fernsehen denken, die Sie sich besonders gerne anschauen: Warum gefallen die Ihnen so gut?"

52 Z. B.: „Damit mir diese Sendungen gefallen, ist es besonders wichtig, ... dass das Gezeigte für mich und mein Leben relevant ist; ... dass ich dabei etwas lerne; ... dass sie ein „Happy End“ haben; ... dass sie spannend sind; ... dass sie witzig und humorvoll sind; ... dass nicht nur die guten Seiten des Lebens gezeigt werden“. 
Dennoch wurde er nur auf den Kategorien „negative Emotionen“ und „Abwesenheit positiver Emotionen“ eingestuft, da nur sie besonders herausstechende, charakteristische Merkmale des Films darstellen. Das Ergebnis dieser Kategorisierung ist in Tabelle 2 dargestellt, indem alle überdurchschnittlichen Ausprägungen grau hinterlegt wurden.

Gemäß der Hypothese sollten sich jeweils vor allem jene Personen gut unterhalten, die Präferenzen für die dominanten Merkmale der Filme äußerten. Um dies zu überprüfen, wurden Korrelationskoeffizienten zwischen den jeweiligen Präferenzen und dem Unterhaltungserleben für jede der vier Filmgruppen berechnet. In Tabelle 2 sind alle signifikanten Korrelationen eingetragen.

Tabelle 2: Dominante Merkmale* der Stimuli und die Korrelation** zwischen Unterhaltungserleben und Präferenz für bestimmte Programmmerkmale

\begin{tabular}{l|c|c|c|c|c|c|}
\hline & $\begin{array}{c}\text { Relevanz/ } \\
\text { Nähe }\end{array}$ & Dynamik & Qualität & Humor & $\begin{array}{c}\text { negative } \\
\text { Emotionen }\end{array}$ & $\begin{array}{c}\text { positive } \\
\text { Emotionen }\end{array}$ \\
\hline $\begin{array}{l}\text { Grosse Pointe } \\
\text { Blank }\end{array}$ & & .26 & & .21 & & .22 \\
\hline Funny Games & & & & & & .25 \\
\hline $\begin{array}{l}\text { Zwilling \& } \\
\text { Zuckermann }\end{array}$ & 28 & & & .21 & & \\
\hline The Cell & & .24 & & & .25 & \\
\hline
\end{tabular}

* Überdurchschnittliche Ausprägungen eines Merkmals bei einem Film sind grau hinterlegt.

* Korrelation nach Pearson. Es sind nur Korrelationen dargestellt, welche auf 95-prozentigem Niveau signifikant sind (1-seitig). Vorzeichen sind nicht dargestellt.

Mit kleinen Abstrichen kann Hypothese 4 bestätigt werden. Bei den beiden Spielfilmen „Grosse Pointe Blank“ und „The Cell“ sind es genau (und nur) die dominanten Filmmerkmale, welche das Unterhaltungserleben bei Personen mit Präferenzen für genau diese Merkmale förderten und bei Personen ohne diese Präferenz minderten. ${ }^{53}$ Bei den beiden europäischen Produktionen ist das Ergebnis nicht ganz so eindeutig. Lediglich die Vorlieben für die Darstellung positiver, angenehmer Themen und Emotionen stehen bei „Funny Games“ im Zusammenhang mit der Höhe des Unterhaltungserlebens. Bei der Dokumentation „Zuckermann und Zwilling“ korreliert wie erwartet die Präferenz für Inhalte mit hoher subjektiver Relevanz und Realismus. Allerdings spielt hier auch die Präferenz für humorvolle und kuriose Themen und Darstellungsformen eine Rolle.

Relevante Personenmerkmale, wie z. B. „passende“ Genrepräferenzen, fördern also im Sinne eines triadischen Fittings (Früh 2002: 142ff.) das Unterhaltungserleben, fehlen sie, wird es gemindert. Also können wir auch aufgrund der Prüfung von Hypothese 4

53 Die kausale Interpretation der Korrelationen wird hier ausschließlich aus Gründen der Anschaulichkeit gewählt. Sie ist aber theoretisch nicht gefordert und wurde deshalb empirisch auch nicht abgesichert. 
davon ausgehen, ein valides Messinstrument für Unterhaltungserleben vorliegen zu haben.

Divergente Validität: Abschließend soll nun noch geprüft werden, inwiefern sich das Konstrukt Unterhaltungserleben bzw. dessen Operationalisierung von anderen Konstrukten und deren Operationalisierungen unterscheidet. Dies stellt nicht nur ein Kriterium für (divergente) Validität dar, sondern ist auch aus forschungsökonomischen Gründen wichtig: Nur wenn unser Unterhaltungsindex zu anderen (und aus unserer theoretischen Perspektive besseren) Ergebnissen führt, ist es sinnvoll und gerechtfertigt, ein neues und vielleicht etwas komplexeres Messinstrument einzuführen.

$H_{5}:$ Es besteht nur ein geringer bis mäßig starker Zusammenhang zwischen dem

Unterhaltungserleben und der Benennung als "Unterhaltung “ durch die Rezipienten.

In allen drei Studien wurde innerhalb der Adjektivliste bzw. der Liste der semantischen Differentiale erfasst, wie stark die Bezeichnung „unterhalten - nicht unterhalten“ (Studie 1 \& 2) bzw. nur „unterhalten“ (Studie 3) auf das „allgemeine Gefühl beim Anschauen des Films“ zutrifft. Zur Prüfung der Hypothese wurden die Korrelationen zwischen „unterhaltend“ und unserem Unterhaltungsindex sowohl für jede Studie als auch für jeden einzelnen Stimulus berechnet (Abbildung 3). Es zeigen sich durchweg signifikante mittlere Zusammenhänge. Der höchste $(\mathrm{r}=0.65)$ besteht in Studie 3 („Unterhaltungsprozess") bei den beiden Hollywood-Produktionen, der geringste in Studie 2 ("Alltagsunterhaltung“) zwischen Nachrichten $(\mathrm{r}=0.35)$ und der „ernsten“ Variante der Dokumentation „Herr Zwilling und Frau Zuckermann“ ( $r=0.37$ ) aus Studie 1. Die Hypothese kann also soweit als bestätigt gelten: Da beide Konstrukte (Unterhaltungserleben und „unterhaltend“) sehr ähnlich sind, gibt es plausiblerweise einen klaren Zusammenhang zwischen beiden Messungen. Dieser ist aber bei weitem nicht so stark, dass wir nicht auch empirisch begründet von unterschiedlichen Konstrukten ausgehen können.

\section{Abbildung 3: Zusammenhang zwischen dem Unterhaltungsindex und der Verwendung} des Labels „unterbaltend“ in den drei Studien bei verschiedenen Stimuli

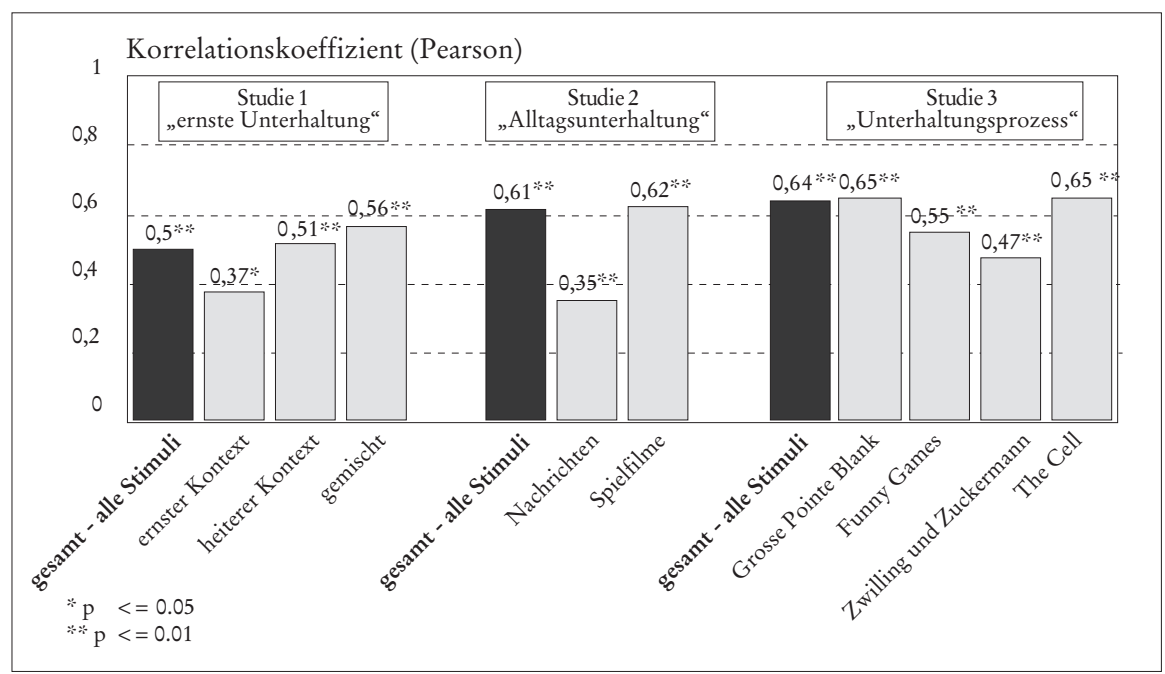


Darüber hinaus ist zu erkennen, dass der Zusammenhang bei gemeinhin als „Unterhaltung“ gelabelten Angeboten („Hollywood-Spielfilme“) stärker ist als bei Inhalten, welche eher einem ernsten Kontext zugeordnet sind (Dokumentationen, Nachrichten). Insbesondere bei diesen Angeboten, die gemeinhin nicht in das Unterhaltungsraster der Programmzeitschriften und der Rezipienten passen, kommt unser Messinstrument also zu anderen Ergebnissen, was als Indiz für eine größere Unabhängigkeit von einer derartigen sozialisierten Semantik interpretiert werden kann.

\section{Diskussion}

Der TDU-Unterhaltungsindex tritt in seinen Anwendungsmöglichkeiten nur zu der schlichten Frage: „Wie gut haben Sie sich unterhalten?“ in Konkurrenz. Am aussagekräftigsten ist er, wenn die Reaktion auf einen konkreten Stimulus erfasst wird. Mit den bekannten Unschärfen lassen sich damit aber auch die Unterhaltungsimages von Angebotstypen oder Genres erfassen. Die Aussagekraft ist, wie bei jeder empirischen Messung, durch die gewählte Methode begrenzt. Erhebungen, die auf Selbstauskunft der Versuchspersonen beruhen, können immer nur nach Indikatoren fragen, die bewusstseinsfähig sind und im Zuge der Verbalisierung einen kognitiven Interpretationsprozess durchlaufen. Da Emotionen jedoch zum großen Teil präkognitive Prozesse darstellen, können die verbalisierenden Methoden durch direktere Verfahren ergänzt werden (z. B. physiologische Messmethoden oder CRM). Auch im Rahmen solcher, meist experimenteller Multimethoden-Forschungsdesigns, lässt sich der Index gut einsetzen.

Der TDU-Unterhaltungsindex ist als universelles Standardinstrument konzipiert. Die zentralen Konstruktbestandteile sind lediglich in ihrer minimalsten Form repräsentiert. Dies soll a) universelle Einsatzmöglichkeiten in Feld- und Laborforschung, b) Transparenz und Vergleichbarkeit von Forschungsergebnissen und c) Offenheit für verschiedenste Forschungsinteressen gewährleisten. Das Messinstrument ist insbesondere auf medienvermittelte Unterhaltung zugeschnitten. Weitere Anwendungsmöglichkeiten sind wahrscheinlich, aber noch nicht im Detail überprüft. Eine solche eventuelle Erweiterung und Generalisierung würde jedoch in erster Linie die zugrunde liegende Theorie betreffen, erst dann können Folgen für das Erhebungsinstrument sinnvoll diskutiert werden. Das Messinstrument bezieht sich zwar auf eine einzige Theorie, da die TDU jedoch als Rahmentheorie konzipiert ist (Früh 2003b), lassen sich viele Theorien, die sich auf einzelne Teilaspekte von Unterhaltung beziehen (z. B. Empathie, parasoziale Interaktion, Spannung etc.), dort entweder als Ausdifferenzierungen allgemein konzipierter TDU-Dimensionen oder aber als eigenständige Module an passender Stelle einfügen. Für den TDU-Index hätte dies zur Konsequenz, dass er nur für die jeweilige spezielle Anwendung (d. h. temporär) durch weitere Skalen systematisch erweitert wird. Allerdings möchten wir vorschlagen, dies als separates Erhebungsmodul zu konzipieren und um der besseren Vergleichbarkeit willen beide Werte einzeln auszuweisen.

Die Modifikationsmöglichkeit kann auch dann genutzt werden, wenn die hier vorgeschlagene Kurzform des Messinstruments für bestimmte Fragestellungen insgesamt als zu undifferenziert erscheint. Wir erwähnten bereits, dass die vorliegende Kurzform auf der Grundlage elaborierterer Instrumente entstand. Dennoch sind wir uns noch nicht ganz schlüssig darüber, wie sinnvoll es ist, daraus einen „Langform-Prototyp“ zu entwickeln, weil die Forschungsinteressen so vielfältig sind, dass auch er immer auf das jeweilige Forschungsziel hin modifiziert und ergänzt werden müsste. Sinnvoller erscheint es vielleicht zu sein, die Kurzform jeweils als Grundgerüst von Dimensionen zu 
benutzen, die darin enthaltenen Kriterien mit ihren Indikatoren zu übernehmen und dann auf das eigene Forschungsziel hin zu ergänzen.

Aus methodischer Sicht halten wir den vorliegenden Index für einen nicht weiter reduzierbaren Prototyp, der sich jetzt in der Forschung weiter bewähren muss. Sehr hilfreich wäre es dabei, wenn das etwas unscharfe bzw. hinsichtlich des betreffenden Teilkonstrukts unvollständige Adjektiv „verträumt“ durch ein (einzelnes!) treffenderes ersetzt werden könnte (Erläuterungen s. o.). Mit „bewähren“ meinen wir zwar auch die Aufdeckung von Bezügen zu anderen, unterhaltungsrelevanten Konstrukten, viel spannender erscheint uns jedoch die Frage, ob das Instrument z. B. tatsächlich (wie postuliert) in allen denkbaren Kontexten, also auch bei den vielen so genannten „nicht-unterhaltenden Genres“ zu aussagekräftigen Ergebnissen führt. Wir meinen, dass viel Erkenntnis- bzw. Erklärungspotenzial verschenkt wird, wenn die Forschung meint, die Variable „Unterhaltung“ sei nur im Zusammenhang mit „typischen Unterhaltungsangeboten“ relevant. Wichtig erscheint auch noch die Klärung der Frage, ob die charakteristischen „Tönungen“ von Unterhaltungserleben, wie z. B. anlässlich von Krimis, Komödien, Historien- oder Natur-Dokumentationen, Nachrichten oder Bildungsangeboten, durch unsere, auf möglichst hoher Abstraktionsebene formulierten Items so abgebildet werden, dass keine systematischen Verzerrungen zugunsten einzelner „Tönungen " entstehen. Dies war zwar Gegenstand unserer Validierungsstudien, aber es handelt sich dabei doch nur um eine einmalige Bestätigung, die an vergleichbaren Stimuli mehrfach bestätigt werden sollte. Wäre dies nicht der Fall, müsste man die vorliegende Minimalversion ggf. durch weitere Items ergänzen, was dann aber auf Kosten der Praktikabilität ginge.

\section{Literatur}

Biocca, Frank A.; Prabu David \& Mark West (1994): Continuous Response Measurement (CRM): A Computerized Tool for Research on the Cognitive Processing of Communication Messages. In: Lang, Annie (Hrsg.): Measuring Psychological Response to Media. Hillsdale: Lawrence Erlbaum, S. 15-64.

Bortz, Jürgen; Nicola Döring (2002): Forschungsmethoden und Evaluation. Berlin, Heidelberg, New York, Tokyo: Springer Lehrbuchverlag.

Csikszentmihalyi, Mihaly (2000): Das flow-Erlebnis. Jenseits von Angst und Langeweile: im Tun aufgehen. Stuttgart: Klett-Cotta.

Debus, Günter (2000): Sprachliche Methoden. In: Otto, Jürgen H.; Harald A. Euler; Heinz Mandl (Hrsg): Emotionspsychologie. Ein Handbuch. Weinheim, Basel: Beltz, S. 409-418.

Früh, Werner (Hrsg.) (2002): Unterhaltung durch das Fernsehen. Eine molare Theorie. Konstanz: UVK.

Früh, Werner (2003a): Triadisch-Dynamische Unterhaltungstheorie (TDU). In: Früh, Werner; Hans-Jörg Stiehler (Hrsg): Theorie der Unterhaltung. Ein interdisziplinärer Diskurs. Köln: Herbert von Halem, S. 27-56.

Früh, Werner (2003b): Theorien, theoretische Modelle und Rahmentheorien. Eine Einleitung. In: Früh, Werner; Hans-Jörg Stiehler (Hrsg.): Theorie der Unterhaltung. Ein interdisziplinärer Diskurs. Köln: Herbert von Halem, S. 9-26.

Grubitzsch, Siegfried (Hrsg.) (1991): Testtheorie - Testpraxis: Psychologische Tests und Prüfverfahren im kritischen Überblick. Hamburg: Rowohlt Taschenbuch Verlag.

Klopp, Pascal (2003): Kommentierungen beim Unterhaltungserleben und ihr zeitlicher Verlauf. Unveröffentlichte Magisterarbeit. Universität Leipzig.

Lombard, Matthew; Theresa Ditton (1997): At the heart of it all: the concept of presence. In: Journal of Computer Mediated Communication, 3(2). Online erhältlich: http://www.ascusc.org/ jcmc/vol3/issue2/lombard.html. 
Oliver, Mary Beth (1993): Exploring the Paradox of the Enjoyment of Sad Films. In: Human Communication Research (19), S. 315-342.

Roser, C. (1990): Involvement, attention, and perception of message relevance in the Response to persuasive appeals. In: Communication Research (17), S. 571-600.

Roskos-Ewoldsen, David; Beverly Roskos-Ewoldsen \& Francesca R. Dillmann Carpentier (2002): Media Priming: A Synthesis. In: Bryant, Jennings; Dolf Zillmann (Hrsg.): Media Effects. Advances in Theory and Research. Second Edition. Mahwah, New Jersey: Lawrence Erlbaum, S. $97-120$.

Salmon, C. T. (1986): Perspectives on involvement in consumer and communication research. In: Dervin, B.; M. J. Voigt (Hrsg.): Progress in communication sciences (7). Beverly Hills, CA: Sage, S. 243-268.

Schmid, Ingrid; Carsten Wünsch (2001): Definition oder Intuition? Die Konstrukte „Information“ \& „Unterhaltung“ in der empirischen Kommunikationsforschung. In: Wirth, Werner; Edmund Lauf (Hrsg.): Inhaltsanalyse: Perspektiven, Probleme, Potentiale. Köln: Herbert von Halem, S. 31-48.

Schmidt, Siegfried J. (2003): Unterhaltung gibt es nicht. Unterhalten Sie sich gut! Einige philosophische Anmerkungen zum Thema. In: Früh, Werner; Hans-Jörg Stiehler (Hrsg): Theorie der Unterhaltung. Ein interdisziplinärer Diskurs. Köln: Herbert von Halem, S. 324-336.

Schnell, Rainer; Paul Hill \& Elke Esser (1999): Methoden der empirischen Sozialforschung. München: Oldenbourg.

Schnotz, Wolfgang (1988): Textverstehen als Aufbau mentaler Modelle. In: Mandl, Heinz; Hans Spada (Hrsg): Wissenspsychologie. München, Weinheim: Psychologie Verlags Union, S. 299-330.

Van Dijk, Teun A. (1980): Macrostructures. An Interdisciplinary Study of Global Structures in Discourse, Interaction, and Cognition. Hillsdale.

Vitouch, Peter (1993): Fernsehen und Angstbewältigung. Opladen. Westdeutscher Verlag. 


\section{Dokumentation des Erhebungsinstruments}

So, jetzt noch einmal mit Abstand betrachtet: Was empfanden oder fühlten Sie so im Großen und Ganzen beim Anschauen des Beitrags (Films, Sendung, Talkshow etc.)? Beschreiben Sie Ihren Eindruck ganz allgemein, ohne jetzt an bestimmte Einzelheiten zu denken.

Dazu haben wir Ihnen eine Liste mit Wörtern vorbereitet, mit denen man das beschreiben kann. Gehen Sie also einfach diese Wörter der Reihe nach durch und entscheiden Sie jedes Mal, wie stark das Wort auf Ihr allgemeines Gefühl beim Anschauen des Beitrags (Films, Sendung, Talkshow etc.) zutrifft.

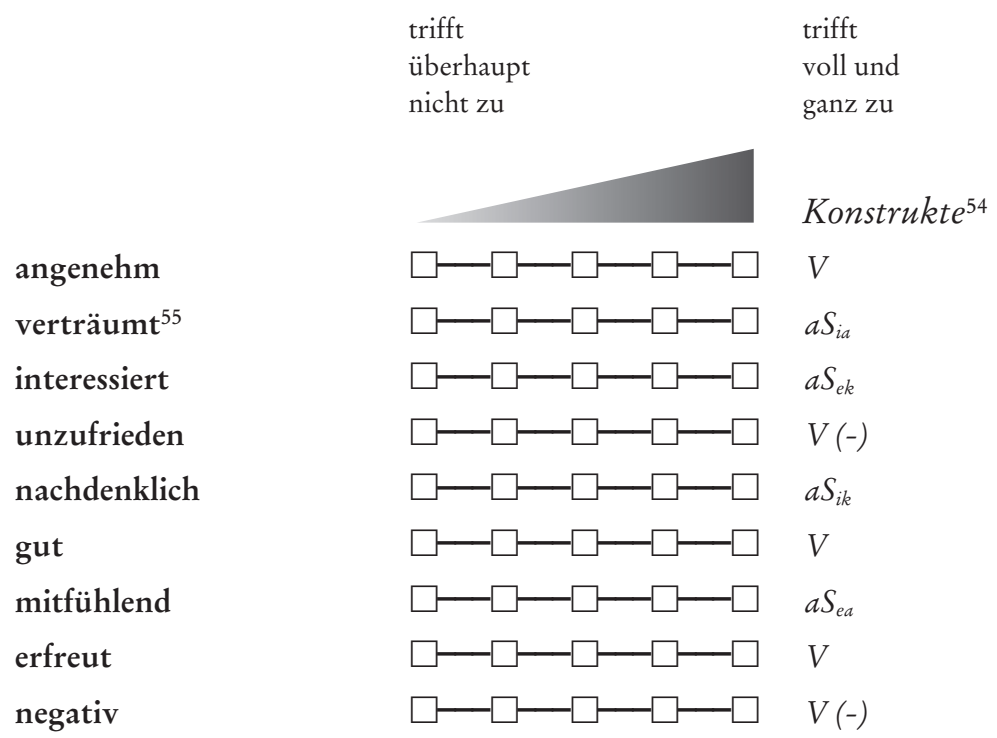

Im Folgenden finden Sie einige Aussagen über den Beitrag (Film, Sendung, Talkshow etc.), den sie gerade gesehen haben.

Gehen Sie bitte einfach die Aussagen der Reihe nach durch und überlegen Sie jedes Mal, wie sehr die Aussagen Ihrer Meinung nach zutreffen.

Kreuzen Sie ganz links an, wenn die Aussage überhaupt nicht zutrifft und ganz rechts, wenn sie voll und ganz zutrifft. Mit den Werten dazwischen können Sie Ihr Urteil abstufen.

54 Legende: siehe FN 25 - Abschnitt 2.2; mit „(-)“ versehene Items sind vor der Indexberechnung umzupolen.

55 Siehe FN 22. 


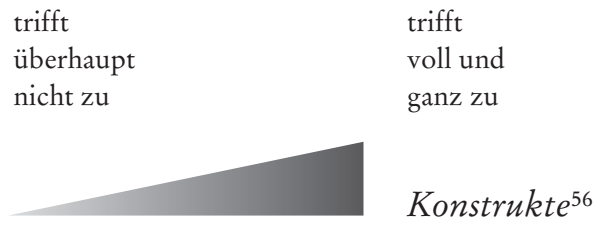

Das Gezeigte betrifft mich

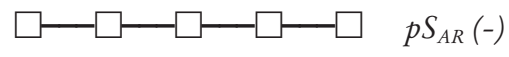
persönlich in meinem Alltag.

Ich hätte eigentlich viel lieber etwas ganz anderes gemacht.

Ich konnte abschalten und den ganzen Alltag mit Familie, Schule oder Beruf vergessen.

Manchmal konnte ich den Beitrag (Film, Sendung, Talkshow etc.) nicht mehr richtig genießen, weil mich das

Gezeigte abgestoßen hat.

Das Gezeigte ist wichtig für mein normales Leben.
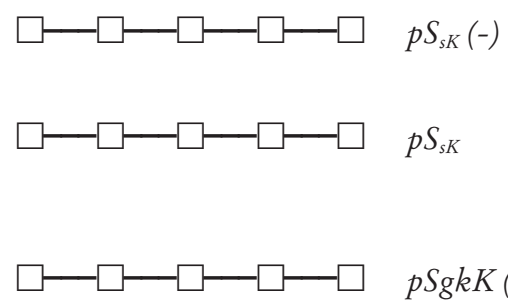

$p S g k K(-)$

\section{Berechnungsvorschrift für den standardisierten Unterhaltungsindex $\left(\mathrm{UI}_{100}\right)$}

1) Alle mit (-) gekennzeichneten Items umpolen und sicherstellen (d. h. kontrollieren und ggf. umcodieren), dass alle Antwortausprägungen mit den Werten 0 bis 4 codiert wurden.

2) Den Valenzindex erstellen: Das arithmetische Mittel aus allen fünf Items zur Valenz berechnen.

3) Den Index „passive Souveränität - Alltagsrelevanz“ erstellen: Das arithmetische Mittel aus den beiden Items zur „passiven Souveränität - Alltagsrelevanz“ berechnen.

4) Den Index „passive Souveränität - situativer Kontext“ erstellen: Das arithmetische Mittel aus den beiden Items zur „passiven Souveränität - situativer Kontext“ berechnen.

5) Den Index „passive Souveränität“ erstellen: Das arithmetische Mittel aus dem Item zur „passiven Souveränität - gesellschaftlich-kultureller Kontext“, dem Index „passive Souveränität Alltagsrelevanz“ und dem Index „passive Souveränität - situativer Kontext“ berechnen.

6) Den Index „aktive Souveränität“ erstellen: Den Maximalwert aus allen vier Items zu den vier Subdimensionen von „aktiver Souveränität“ ermitteln.

7) Den Unterhaltungsindex (UI) erstellen: Das Produkt aus den Indizes „Valenz“, „passive Souveränität" und „aktive Souveränität" berechnen.

8) Den standardisierten Unterhaltungsindex $\left(\mathrm{UI}_{100}\right)$ erstellen: Den Unterhaltungsindex durch $64^{57}$ dividieren.

56 Legende: siehe FN 25 - Abschnitt 2.2; mit „(-)“ versehene Items sind vor der Indexberechnung umzupolen.

57 Dies entspricht dem Produkt der Skalenendpunkte aller drei unter 7) verwendeten Indizes. 